\title{
Photoactivation of Cryptochromes Invokes
}

\section{Competing Inter- and Intramolecular Electron}

\section{Transfer}

\author{
Ruslan N. Tazhigulov, ${ }^{\dagger, \dagger}$ Justin Provazza, ${ }^{\dagger}$ David F. Coker, ${ }^{\dagger}$ and Ksenia B. \\ Bravaya*, \\ $\dagger$ Department of Chemistry, Boston University, Boston, Massachusetts 02215, United States \\ $\ddagger$ Division of Chemistry and Chemical Engineering, California Institute of Technology, \\ Pasadena, California 91125, United States \\ E-mail: bravaya@bu.edu
}

\begin{abstract}
Growing experimental and theoretical evidence points to the key role of cryptochrome proteins in magnetoreception by migratory birds and insects. Cryptochrome photoactivation is achieved through a cascade of electron transfer events leading to formation of a long-lived spin-correlated radical pair. The electron transfer cascade is initiated by photoexcitation of the FAD cofactor and subsequent electron transfer through three conserved tryptophan residues, the so-called tryptophan triad. Presence of ATP was shown to increase the yield of the semireduced form of FAD. While electron transfer through the tryptophan triad is well characterized by both theoretical and experimental methods, the effects of ATP binding are still not well understood. The present work aims to unravel the mechanism of ultrafast photoinduced electron transfer in a cryptochrome protein with a focus on effects of ATP on the FAD photoreduction
\end{abstract}


process. Photoinduced electron transfer is described by means of state-of-the-art theoretical methods: a hybrid quantum-classical polarizable embedding scheme is utilized to accurately parameterize a generalized local excited/charge transfer state system-bath model Hamiltonian and the photoinduced electron transfer process is described by a semiclassical path integral-based dynamics method. The results draw attention to the crucial role of the intramolecular electron transfer from adenine to the flavin moiety of the FAD cofactor for formation of the semireduced form of FAD, providing an explanation for the increased yield of the semireduced form in the presence of the cellular metabolites in vitro and in vivo. 


\section{Introduction}

Photoinduced electron transfer (PET) processes are at the core of energy conversion in biological systems and artifical biomimetic devices. ${ }^{1-8}$ In living organisms, light absorption is often followed by a cascade of energy and/or charge transfer (CT) events that initiate energy conversion and signal transduction. ${ }^{9-11}$ A first-principles description of such processes can provide valuable physical insights, and help to unravel the underlying molecular mechanisms. The new knowledge obtained from advanced computational and experimental studies can further be used to guide development of biomimetic devices ${ }^{8}$ such as, for example, artificial flavoprotein magnetosensors. ${ }^{7}$ Development of such artificial protein maquettes is inspired by the extensive experimental and theoretical studies on the natural magnetic compass system in migratory birds. The magnetic compass is supposedly mediated by cryptochrome proteins located in bird retinas. ${ }^{5}$ Multiple in vitro experimental and in silico computational studies have aided in determining the general mechanism of intraprotein electron flow and its potential relevance to magnetosensory function. However, mechanistic features of the ultrafast PET in cryptochromes, representing a crucial step of the cryptochrome photoactivation process, as well as modulation of the ultrafast PET through interactions with cellular metabolites in vivo remain unclear.

Cryptochromes are flavoproteins playing various functional roles in living species. They are involved in growth of plants, participate in circadian clock machinery of plants and animals, and repair single-stranded DNAs (for the so-called DASH, Drosophila, Arabidopsis, Synechocystis, Homo, cryptochromes) similar to their evolutionary ancestors, photolyases. ${ }^{5,12,13}$ Interestingly, Ahmad and coworkers recently reported that exposure of cells with the human cryptochrome to a weak pulsed electromagnetic field leads to rapid accumulation of reactive oxygen species (ROS), a potentially toxic metabolite that plays a role in cellular aging and stress response. ${ }^{14}$ Based on this report, Keays and coworkers suggested that childhood leukemia, associated with low-intensity magnetic fields produced by power lines, and animal magnetoreception could be linked. ${ }^{15}$ Growing experimental evidence also points to the 
key role of cryptochromes as the biological magnetosensors in migratory birds and insects. While cryptochromes can be photoreceptors associated with magnetosensory function, the identity of the primary signaling biological system that interacts with the geomagnetic field and the relevant magnetosensitive reaction is still a subject of ongoing research. ${ }^{5,16}$ One of the mechanisms that can explain magnetosensitivity was originally proposed by Schulten et al. ${ }^{17}$ The mechanism implies formation of a relatively long-lived spin-correlated radical pair. The chemical nature of the primary signaling radical pair in this mechanism is still under debate. ${ }^{18-29}$

In the dark, flavin of the FAD cofactor in plant cryptochromes exists in the fully oxidized ground state. ${ }^{30-33}$ Photoexcitation of the FAD cofactor initiates a cascade of ET reactions involving three evolutionarily conserved tryptophan residues, ${ }^{34-37}$ and leads to formation of the semireduced form of FAD. Upon the blue-light photoexcitation of the flavin chromophore, the first electron transfer occurs from the tryptophan W400 (Fig. 1) to the FAD flavin. The hole then propagates to the protein surface through the second W377, and third W324 tryptophans (Fig. 1A). W400, W377 and W324 in a plant cryptochrome from Arabidopsis thaliana form the so-called tryptophan triad. The driving force for the hole propagation was attributed to increasingly more extensive solvation of the tryptophan radical cations along the W400-W377-W324 triad. ${ }^{38}$ Interestingly, plant and animal cryptochromes were shown to have a different terminal hole acceptor. In contrast to plant cryptochromes, the fourth terminal tryptophan, W394, was proposed as a subsequent hole acceptor in the Drosophila melanogaster animal cryptochrome based on the amino acid sequence similarities with (6-4) photolyases. ${ }^{39}$ Formation of $\mathrm{W}_{394^{+}}$was supported experimentally by transient electron-paramagnetic resonance and transient optical spectroscopy. ${ }^{40}$

Moreover, a tyrosine residue Y373, structurally homologous to the fourth tryptophan W394 in Drosophila melanogaster cryptochrome, was shown to be a terminal hole acceptor in the Chlamydomonas reinhardtii plant cryptochrome. ${ }^{40}$ The spatially separated radical pair $\left(\mathrm{Flv}_{\mathrm{V}} \operatorname{Trp}_{3} / \operatorname{Trp}_{4}\right)$ is long-lived with a lifetime in the ms range, ${ }^{30,40-42}$ and was initially 
proposed to be the one that is able to interact with the magnetic field. ${ }^{18,19,21}$ However, other candidates for the radical pair were also proposed recently. ${ }^{27}$ The species that have been discussed as alternative second radical sites in the spin-correlated radical pair include: superoxide $\mathrm{O}_{2}{ }^{-}$, dioxygen $\mathrm{O}_{2},{ }^{22-24,26}$ hydroperoxyl radical $\mathrm{HO}_{2},{ }^{27}$ tyrosyl radical, ${ }^{27}$ a metal ion, ${ }^{27}$ ascorbyl radical, ${ }^{27}$ or ligands that can be potentially bound at the position of the DNAbinding site in structurally similar photolyases. ${ }^{12,27}$ Very fast electron spin relaxation, ${ }^{22,43}$ very strong anisotropic hyperfine interactions for hydroperoxyl radical $(\approx 1 \mathrm{mT}),{ }^{27,44}$ and no experimentally confirmed $\mathrm{O}_{2}$-binding site in cryptochromes make superoxide, dioxygen, and hydroperoxyl radical unlikely candidates for the signaling radical pair, ${ }^{27}$ though recent theoretical investigations reveal that the Drosophila melanogaster cryptochrome has a potential to bind them in the close proximity to the flavin of FAD. ${ }^{45}$ The tyrosyl radical, for which the hyperfine couplings are as strong as for the tryptophan radical, ${ }^{27,46}$ is experimentally found to be formed on the microsecond timescale in the plant cryptochrome. ${ }^{41}$ Moreover, it has been recently reported that the tyrosine Y373 serves as the fourth electron donor in the $\mathrm{ET} /$ proton-coupled ET chain in the cryptochrome from Chlamydomonas reinhardtii with a radical pair lifetime of $\approx 50 \mathrm{~ms} .{ }^{47}$ Therefore, despite thorough and extensive theoretical and experimental studies of cryptochromes and their homologs, mechanistic features of the PET specifically for cryptochromes are still missing. The present work revisits and sheds new light on the role of FAD itself, and cellular metabolites, in photoactivation of cryptochromes.

The intramolecular ET from adenine to the flavin of FAD was observed experimentally for its stacked conformation in water solution. The measured time constant is $\approx 4$ ps for the ET initiated from the lowest excited singlet state $\mathrm{S}_{1}$ (referred to as LE in the present work, see Fig. 1B). ${ }^{48-51}$ A slightly faster $(\approx 1.1 \mathrm{ps})$ intramolecular ET has been reported for $\mathrm{FAD}$ in $\mathrm{D}_{2} \mathrm{O} .{ }^{52}$ Moreover, the intramolecular ET between adenine and flavin of FAD has been extensively explored in the work on evolutionary ancestors of cryptochromes, the photolyases, by Zhong and coworkers. ${ }^{53-55}$ The reported time constant for the intramolecular ET in the mutated photolyase (E109A/W382F/W384F, effectively blocking the tryptophan 

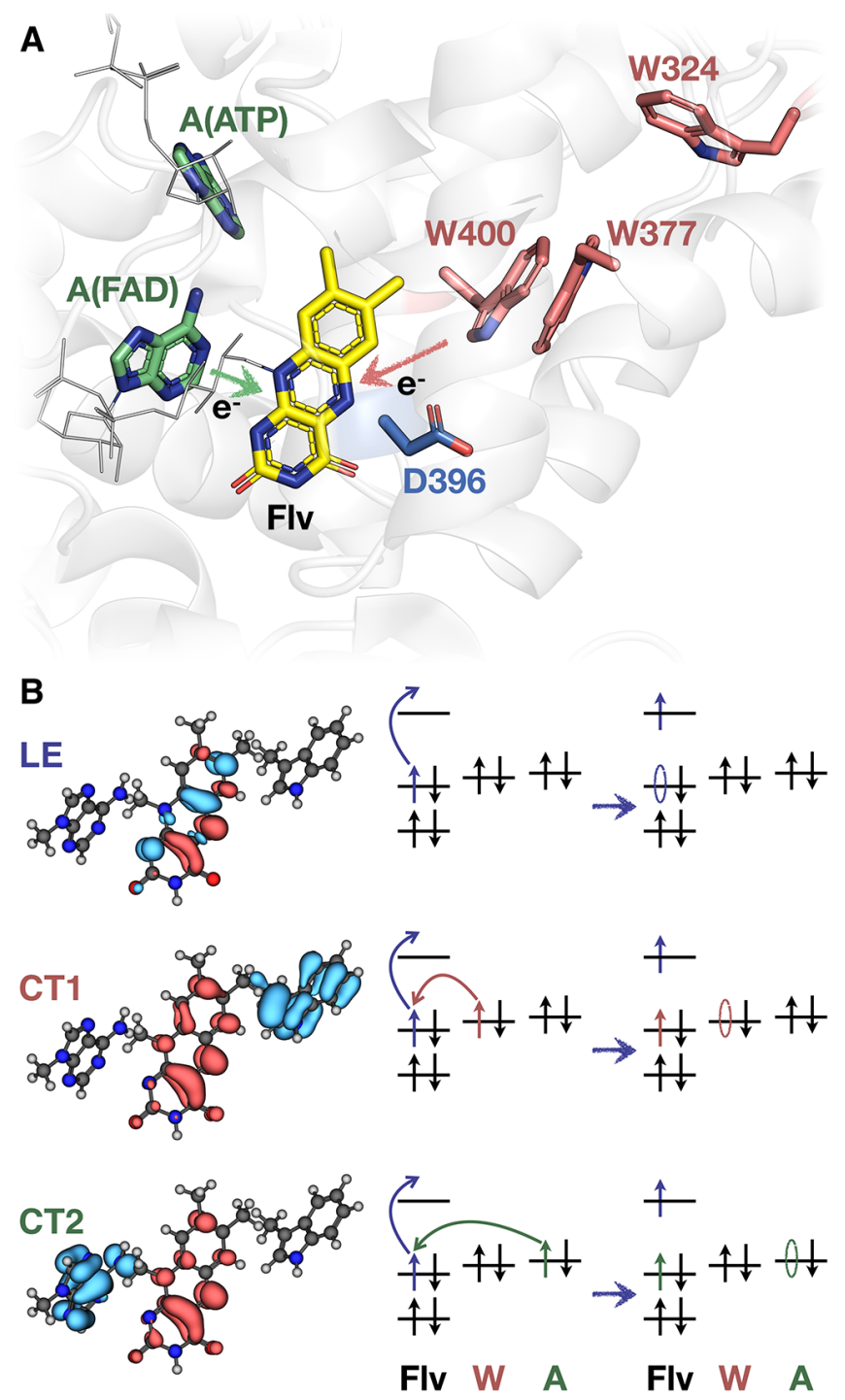

Figure 1: (A) Photoactive site of Cry1At-PHR. Two potential electron transfer pathways initiated by blue-light photoexcitation of the flavin moiety (Flv) of FAD are shown: from W400 and from adenine of FAD (A(FAD)). (B) Difference densities w.r.t to the ground state for locally excited (LE), charge transfer 1 (CT1, with the hole on W400), and charge transfer 2 (CT2, with the hole on $\mathrm{A}(\mathrm{FAD})$ ) states. 
triad and other ET channels) is 19 ps, and the time constant for the backward process (from the charge transfer state back to the ground state) is $100 \mathrm{ps}$. In the same studies, a $0.8 \mathrm{ps}$ time constant has been assigned for the ET from the nearest tryptophan to flavin. ${ }^{53-55}$ As for the experimental time constants for ET in other flavoproteins, similar processes occur in glucose oxidase ${ }^{56}$ and BLUF proteins. ${ }^{57}$ However, the FAD cofactor in glucose oxidase is present in the open conformation, as opposed to the stacked one in cryptochromes and photolyases, preventing any intramolecular ET between adenine and flavin. The ultrafast ET between flavin and tryptophan/tyrosines in glucose oxidase occurs at 1.8 ps. ${ }^{56}$ Much slower ET from the tyrosine to flavin $(\approx 7 \mathrm{ps})$ has been observed in BLUF proteins. ${ }^{57}$ While it has been extensively studied in photolyases, to the best of our knowledge, there is no conclusive experimental data on the existence of, or a time scale for, the intramolecular ET from adenine of FAD to flavin in cryptochromes (Fig. 1).

Another question is whether the PET in cryptochromes is modulated in the presence of cellular metabolites, or whether the mechanism of photoactivation can be different in vivo, when they are exposed to cellular metabolites. Binding of cellular metabolites by cryptochromes, in particular of ATP, was shown to affect multiple aspects of cryptochrome photochemical activity. It was linked to enabling autophosphorylation, ${ }^{58}$ initiating conformational changes ${ }^{59,60}$ slowing down the reoxidation process, ${ }^{59}$ shifting $\mathrm{p} K_{\mathrm{a}}$ of the aspartic acid D396, ${ }^{61,62}$ which is a putative proton donor to $\mathrm{FAD}^{--}$, favoring a reaction pathway yielding long-lived aspartate D396-, and increasing levels of FADH· product. ${ }^{59,63,64}$ Importantly, bound ATP was discussed as a potential hole acceptor in another (additional to the tryptophan triad) ET pathway. ${ }^{63,64}$

Here, we explore the PET in a cryptochrome protein. In order to describe this system from first principles, one needs to develop a computational protocol, which can accurately treat the electronic structure of its large photoactive site, as well as properly incorporate protein-solvent environment interactions. In this work, we used a protocol based on constructing the model Hamiltonian for describing the PET in a cryptochrome protein. The 
model Hamiltonian approach has been widely used to characterize energy and charge transfer in a variety of the biological systems. ${ }^{65-73}$ It provides a clear computational advantage over ab initio nonadiabatic simulations (Ehrenfest or surface hopping), ${ }^{74,75}$ considering the large system size, and involvement of multiple electronic states. Moreover, the model Hamiltonian approach, combined with the appropriate quantum dynamics methods, can account for quantum (de)coherence ${ }^{76}$ and other dynamical effects. Model Hamiltonians have been extensively used to characterize energy transfer in photosynthetic light harvesting systems. The parameters of the model Hamiltonians can be obtained either by fitting to the experimental data, ${ }^{65-68}$ or from electronic structure calculations. ${ }^{69-73}$ The latter approach has been previously used in combination with a semiclassical path integral dynamics method known as the partial linearized density matrix (PLDM) ${ }^{77-79}$ propagation scheme, for simulating the energy transfer in light harvesting photosynthetic complexes.

The effects of the environment on the electronic structure of the chromophores are taken into account by using the electronic embedding quantum mechanics/molecular mechanics $(\mathrm{QM} / \mathrm{MM})$ scheme, which accounts for polarization of the QM part by the MM part, but neglects the reverse polarization. Our previous studies on redox potentials of a cryptochrome protein showed that such effects can be significant: the polarization effects increase the vertical ionization/attachment energies and corresponding redox potentials by up to $1.7 \mathrm{eV}$ and $1.4 \mathrm{~V}$, respectively. ${ }^{80}$ Similarly, one can expect that the polarization effects can be crucial for an accurate description of charge separation processes owing to the significant charge density redistribution upon electronic transition. In this work, we exploited hybrid polarizable embedding QM/MM techniques that account for the environment polarization together with the nonadiabatic semiclassical dynamics method to explore PET in the photolyase-homologous region of cryptochrome 1 from Arabidopsis thaliana (Cry1At-PHR).

Therefore, the goals of the present work are two-fold: we aim (1) to explore the relative efficiency of the intramolecular ET pathway from adenine of FAD to flavin and the ET pathway from tryptophan W400 to flavin, and (2) to explain the role of ATP on the increased 
yield of the semireduced form of FAD, which has been observed experimentally. ${ }^{63,64}$

In addition, we discuss the role of polarization effects, and of the electronic-nuclear couplings in driving PET in cryptochromes. We provide theoretical estimates of the rate constants and quantum yields and discuss their relation to the available experimental data on cryptochromes from Chlamydomonas reinhardtii ${ }^{81}$ and Arabidopsis thaliana. ${ }^{61}$

\section{Methods}

Model Hamiltonian. The model Hamiltonian describing the interactions of the electronic subsystem with the bath of nuclear degrees of freedom has the following form:

$$
\begin{aligned}
\hat{H}(Q)= & \sum_{A} \epsilon_{A}\left(Q_{0}\right)|A\rangle\left\langle A\left|+\sum_{A \neq B} \Delta_{A B}\left(Q_{0}\right)\right| A\right\rangle\langle B| \\
& +\sum_{A} \sum_{i} c_{i}^{(A)} \hat{Q}_{i}^{(A)}|A\rangle\langle A|+\sum_{A} \sum_{i} \frac{1}{2}\left(\omega_{i}^{2(A)} \hat{Q}_{i}^{2(A)}+\hat{P}_{i}^{2(A)}\right)
\end{aligned}
$$

where $\epsilon_{A}\left(Q_{0}\right)$ and $\Delta_{A B}\left(Q_{0}\right)$ are the site energy of electronic state $A$ (vertical excitation energy from the ground state to the locally excited or charge transfer states) and electronic coupling between the state $A$ and state $B$ at the nuclear configuration $Q_{0}\left(Q_{0}\right.$ is the origin of the coordinate system and is set to 0$). c_{i}^{(A)}$ is the bilinear electronic-vibrational coupling between the electronic state $A$ and vibrational mode $i$ in the corresponding nuclear bath. $\hat{Q}_{i}^{(A)}$ and $\hat{P}_{i}^{(A)}$ are coordinate and momentum operators of the vibrational modes associated with the state $A$. In this work, $A$ and $B$ label the electronic states described above (see Fig. 1B): locally excited (LE, $\mathrm{S}_{1}$ state of lumiflavin), charge transfer 1 (CT1, with the hole on tryptophan W400), and charge transfer 2 (CT2, with the hole on adenine of FAD).

We have constructed 50 instantaneous model Hamiltonians corresponding to each independent geometry sampled from the classical molecular mechanics molecular dynamics (MM MD) trajectories. All classical MD simulations were performed with the GROMACS package. ${ }^{82}$ To explore the effects of ATP binding, we used two crystal structures. The initial 
structures for the classical MM MD with and without ATP were obtained from the Protein Data Bank, PDB ID 1U3D and 1U3C, respectively. ${ }^{83}$ The MM MD simulations were performed using the prescription from our previous work, and the simulation details can be found in Ref. 80. The first 5 ns of the production run trajectory were discarded, and 50 snapshots were selected 200 ps apart over the next 10 ns. For each MM MD snapshot, the geometry of the region of interest, which includes lumiflavin (methylated flavin of FAD), 9methyladenine (methylated adenine of FAD), and 3-methylindole (the side chain of W400), was locally optimized within the fixed MM environment, eliminating possible artifacts coming from the MM MD geometries. ${ }^{70,80}$ The local QM/MM optimization was performed at $\omega B 97 X-D / 6-31 G / / M M$ point charge (PC) level. Previous studies on the effects of polarizable force fields on the equilibrium geometries have shown that the effects are negligible for the systems with strong hydrogen bonding or electrostatic interactions, and are small but noticeable for conjugated molecular systems, i.e. retinal chromophore in rhodopsin. ${ }^{84}$ The key details on computation of parameters of the model Hamiltonian are provided below.

Site Energies and Electronic Couplings. Site energies and electronic coupling parameters of the model Hamiltonian (Eq. 1), have been computed using hybrid quantumclassical (QM/MM) methods. The QM part included only the chromophores: methylated flavin, methylated adenine of FAD, and the side chain of W400. The rest of the system has been treated at the MM force field level.

Energies of the locally excited (LE) state were initially computed with scaled-oppositespin configuration interaction singles with a perturbative double correction (SOS-CIS(D)) method $^{85}$ and cc-pVDZ basis. ${ }^{86}$ It has been previously shown that SOS-CIS(D) yields excitation energies for a test set of organic dyes with a $0.1 \mathrm{eV}$ accuracy. ${ }^{87}$ Moreover, as the energies in this work have been initially computed with a relatively small basis set (cc-pVDZ), the additive basis set correction to aug-cc-pVTZ ${ }^{88}$ has been applied to mitigate the basis set dependence. The site energies of charge transfer (CT) states have been calculated using the CDFT-CI approach. ${ }^{89,90}$ The long-range corrected $\omega$ B97X-D functional ${ }^{91,92}$ has been used. 
The energies have been first evaluated with a smaller 6-31G(d) ${ }^{93,94}$ basis. An additive correction to $6-311++\mathrm{G}(\mathrm{d}, \mathrm{p})$ basis ${ }^{95,96}$ has been further applied to the CT state energies, similar to those for the LE state. All electronic structure calculations have been performed using the Q-Chem package. ${ }^{97}$

The effects of the protein-environment on the site energies have been taken into account by exploiting a nonempirical polarizable embedding scheme that is an extension of the effective fragment potential (EFP) ${ }^{98-102}$ approach to macromolecules mEFP/BioEFP. ${ }^{80,103}$ The electrostatic and polarization terms account for most of the effects of the environment on the electronic structure of the QM part, and dominate the environmental contributions to the computed state energies. ${ }^{80,104-110}$ Similar to our previous work, ${ }^{80}$ only QM/EFP and $\mathrm{EFP} / \mathrm{EFP}$ electrostatic and polarization terms have been included. The site energies computed with a non-polarizable embedding, but using BioEFP electrostatics, are referred to as NP-BioEFP.

The electronic couplings have been computed with an electronic embedding using the Generalized Mulliken-Hush (GMH) ${ }^{111}$ approximation at the CIS ${ }^{112}$ level (CIS/6-31+G(d,p)//PC scheme). The advantage of the GMH approach combined with the CIS electronic structure method is in the ability to directly compute electronic couplings between the excited states, in particular between the (locally) excited and charge transfer states.

We compute the couplings using the three-state, as opposed to the standard two-state, approximation, similar to the work by Cave and coworkers. ${ }^{113}$ CDFT-CI ${ }^{89,90}$ can also be used for computation of electronic couplings, however, it enables only the calculation of the electronic couplings between the ground, not excited, and charge transfer states. While previous studies have approximated the excited state-charge transfer state couplings as equal to those between the ground (GS) and CT states, ${ }^{114}$ we have found that in our case the electronic couplings between the ground and CT states are much higher than those between the LE and CT states (Table S2). Our choice of electronic structure method and the scheme for computing couplings is based on the results of our benchmark calculations of the electronic 
couplings computed with the various electronic structure methods (CIS, TD-DFT, CDFT$\mathrm{CI}$ ) and schemes (PC, BioEFP, NP-BioEFP). The corresponding couplings can be found in Table S1, S2 and S3 of the Supporting Information. One can see from Tables S2 and S3 that CIS couplings between the GS and CT states are in good agreement with the CDFTCI values, whereas this is not the case for the TD-DFT results. Therefore, CIS couplings between the LE and CT states have been used in our further studies.

Electronic-Vibrational Couplings. Generalizing the prescription of Lee and Coker, ${ }^{70}$ the electronic-vibrational couplings were computed by splitting the nuclear bath into two components. The intra-fragment nuclear bath is represented by the nuclear degrees of freedom of the QM part. The intermolecular bath is represented by the nuclei of the rest of the protein-solvent environment. The intra-fragment electronic-vibrational couplings, $c_{i}^{(A)}\left(Q_{0}\right)$, were evaluated based on the computed gradients at Franck-Condon (FC) points in the normal mode coordinates. The corresponding intra-fragment spectral density has the following form:

$$
J_{\text {intra }}^{(A)}(\omega)=\frac{\pi}{2} \sum_{i} \frac{c_{i}^{(A) 2}}{\omega_{i}} \delta\left(\omega-\omega_{i}\right)
$$

where $\omega_{i}$ is the ground state vibrational normal mode frequency. The standard assumption underlying this model is that the excited state normal modes and frequencies are the same as those of the ground state and that the excited state surface is just shifted in these coordinates relative to the ground state, and the shift is simply related to the electronic-vibrational couplings and can be computed from the excited state gradients at the FC point. The normal mode frequencies and these excited state gradients have been obtained at the $\omega \mathrm{B} 97 \mathrm{X}-\mathrm{D} / 6$ 31G//PC level. In our generalization, ${ }^{70}$ the intra-fragment electronic-vibrational couplings in addition to the couplings associated with the normal modes on individual chromophores (methylated flavin, methylated adenine of FAD, and the side chain of W400) also include the couplings associated with concerted motion of the three chromophores.

For practical purposes, the $\delta$-functions in Eq. 2 have been replaced with fixed width Lorentzian-type functions, which incorporate broadening by dynamics and nuclear dephas- 
ing $^{70,115}$ (see Sec. S7 of Supporting Information).

Unlike energy transfer, charge transfer processes may involve significant electronic-vibrational couplings corresponding to notable protein-solvent reorganization effects that are induced by large charge redistributions of CT states (see Fig. 1B). The intermolecular spectral densities, accounting for the electronic-vibrational couplings with the rest of the environment, were evaluated using excitation energy fluctuation correlation functions according to the following expression: ${ }^{116}$

$$
J_{\text {inter }}^{(A)}(\omega)=\beta \omega \int_{0}^{\infty} d t\left\langle\delta \epsilon^{(A)}(0) \delta \epsilon^{(A)}(t)\right\rangle \cos (\omega t)
$$

where $\beta=\frac{1}{k_{B} T}$ with Boltzmann constant $k_{B}$ and temperature $T, \omega$ is a frequency for cosine transform. The excitation energy fluctuation in Eq. $3\left(\delta \epsilon^{(A)}(t)\right)$ is dominated by the electrostatic interaction and can therefore be approximated using Coulomb interactions between the chromophore partial charge differences $\left\{\Delta q_{I}^{(A)}\right\}$, obtained from the electronic structure calculations, and the environment point charges $\left\{Z_{J}\right\}$, taken from a standard MM force field: 116

$$
\delta \epsilon^{(A)}(t) \approx \sum_{I J} \frac{\Delta q_{I}^{(A)} Z_{J}}{\left|r_{I}(t)-R_{J}\right|}
$$

where $\Delta q_{I}^{(A)}=q_{I}^{(A)}-q_{I}^{\mathrm{gr}}$ is the difference between the excited state $(A)$ and ground state partial charges; $I$ runs over the nuclear centers of the chromophore, $J$ runs over the nuclear centers of the environment.

For each intra-fragment and intermolecular baths, the reorganization energies have been calculated as follows:

$$
\lambda=\frac{1}{\pi} \int_{0}^{\infty} \mathrm{d} \omega \frac{J(\omega)}{\omega}
$$

\section{$3 \quad$ Results and Discussion}

The results of the semiclassical path integral based PLDM ${ }^{77-79}$ dynamics of PET in Cry1AtPHR using the model Hamiltonian approach are discussed below. First, we focus on charac- 
teristics of the isolated electronic subsystem, and quantify the effects of the ATP binding and environment polarization on the computed site energies (Sec. 3.1). Second, we explore the key features of the spectral densities, representing coupling between the electronic subsystem and the nuclear bath (Sec. 3.2). Finally, we present the results of the dynamical simulations (Sec. 3.3 and 3.4), and discuss the importance of the competing intramolecular pathway in formation of the semireduced FAD in Cry1At-PHR.

\subsection{Electronic Hamiltonian}

In the system-bath Hamiltonian, the system is represented by three electronic states: locally excited $\mathrm{S}_{1}$ (LE, $\left\{\mathrm{Flv}^{*}-\mathrm{W} 400-\right.$ Ade $\left.\}\right)$ and two charge transfer states (Fig. 1). CT1 $\left\{\mathrm{Flv}^{{ }^{-}}{ }_{-}\right.$ W400 ${ }^{+}-$Ade $\}$, in which a hole and an electron reside on W400 and flavin of FAD, respectively, is populated in a first electron transfer step along the main tryptophan triad pathway. CT2 $\left\{\mathrm{Flv}^{-}-\mathrm{W} 400-\mathrm{Ade}^{+}\right\}$with a hole and an electron residing on adenine and flavin of FAD, respectively, represents the first step along the proposed competing electron transfer pathway. The system is initially excited to the LE state and then is allowed to evolve to the CT1 and CT2 states. Below we discuss the computed site energies and electronic couplings, as well as the changes of these quantities upon ATP binding and their dependence on protein environment polarization.

\subsubsection{Effects of ATP binding}

First, the effects of ATP binding on the site energies have been explored. The ATP itself has a negative "-4" charge at neutral pH due to the triphosphate group. However, this negative charge is partially compensated by $\mathrm{Mg}^{2+}$, forming an ion pair with the triphosphate group. Due to proximity of the triphosphate group to the adenine of FAD, one can expect the most significant effects for the CT2 site energies owing to the stabilization of the positive charge on the adenine. Indeed, the presence of ATP lowers the CT2 state energy by $0.26 \mathrm{eV}$ (Fig. 2). Note that the environment polarization slightly counter-balances the effect of ATP presence: 
NP-BioEFP results (non-polarizable environment) show a difference of $0.36 \mathrm{eV}$, which is 0.1 $\mathrm{eV}$ greater than for the BioEFP values. LE and CT1 excitation energies are affected much less and the overall shifts for these states in the presence of ATP are within $0.1 \mathrm{eV}$.

The ensemble-averaged LE-CT2 electronic couplings do not change in the presence of ATP, and the differences are within the margins of statistical uncertainty: $209 \mathrm{~cm}^{-1}$ and $205 \mathrm{~cm}^{-1}$ in the absence and presence of ATP, respectively (Table 1). Therefore, the effect of this change on the dynamics is expected to be negligible. However, importantly, the electronic couplings between the LE and CT1 states increase in the presence of ATP: $88 \mathrm{~cm}^{-1}$ vs. $125 \mathrm{~cm}^{-1}$ for the ensemble-average values. Thus, one can expect the CT1 state to be populated more in the presence of the cellular metabolite. The magnitudes of the couplings are close to the values previously reported by de la Lande and coworkers. ${ }^{62}$ The couplings from Ref. 62, however, were approximated by the ground state (not LE) - charge transfer state values, and our calculations show that those can differ from the LE - charge transfer state ones by up to an order of magnitude (see Table S3 in SI). CT1-CT2 electronic couplings have been found to be much smaller than those for LE-CT1 and LE-CT2 and are not affected much by the binding of ATP (Table 1). 


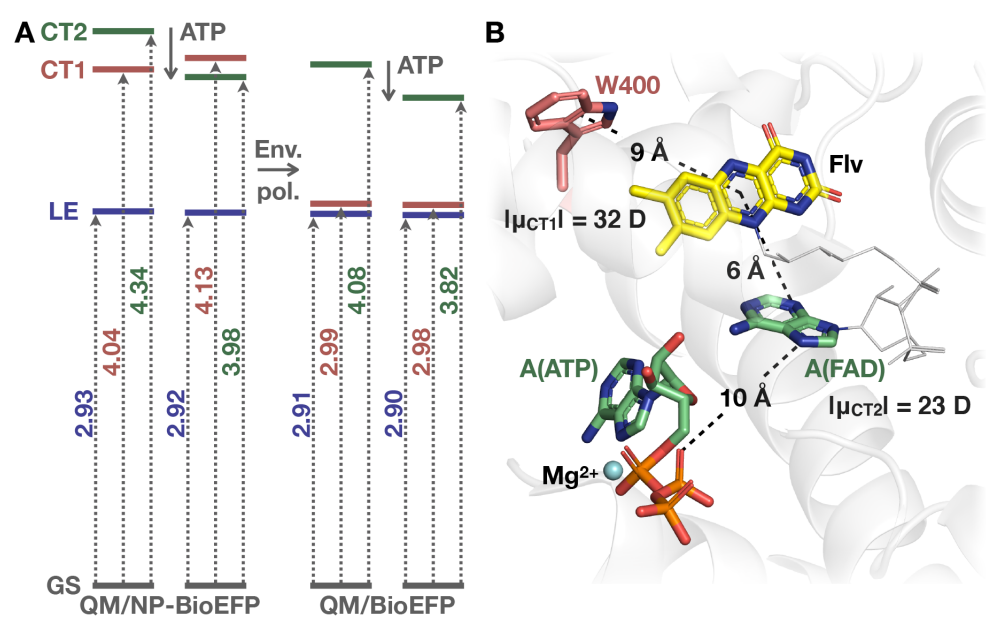

Figure 2: (A) Ensemble-averaged site energies (in eV) computed with SOS-CIS(D)/augcc-pVTZ for LE and CDFT-CI, $\omega$ B97X-D/6-311++G(d,p), for CT1 and CT2 states with non-polarizable (NP-BioEFP) and polarizable (BioEFP) embedding for 50 configurations with and without ATP (see details in Sec. 2). Uncertainty for site energies does not exceed $0.08 \mathrm{eV}$. (B) Distances between the chromophores and the absolute values of diabatic dipole

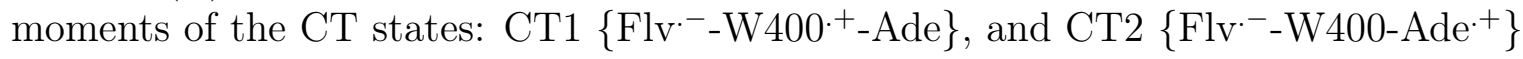

Table 1: Ensemble-averaged unsigned electronic couplings $\left(\mathrm{in}^{-1}\right)$ between LE, CT1, and CT2 states computed with the three-state GMH scheme at CIS level and averaged over 50 configurations with and without ATP

\begin{tabular}{ccc}
\hline \hline & without ATP & with ATP \\
\hline LE-CT1 & $88 \pm 23$ & $125 \pm 36$ \\
LE-CT2 & $209 \pm 51$ & $205 \pm 35$ \\
CT1-CT2 & $40 \pm 28$ & $55 \pm 19$ \\
\hline
\end{tabular}

\subsubsection{Effects of Polarizable Environment}

One can expect the environment polarization to alter the energetics of the charge transfer states owing to significant charge redistribution upon electronic transitions. Similar trends have been observed for vertical ionization and electron attachment energies in the same protein system. ${ }^{80}$ Indeed, the environment polarization significantly lowers the site energies for the charge-transfer excited states: by $1.05-1.15 \mathrm{eV}$ and by $0.16-0.26 \mathrm{eV}$ for the $\mathrm{CT} 1$ and CT2 states, respectively. Note that charge separation occurs with much shorter distances for the CT2 state ( $\approx 6 \AA$ between the centers of mass of the aromatic moieties, Fig. 2 ), 
compared to the CT1 state $(\approx 9 \AA)$. Therefore, one would expect the polarization effects to be smaller for the CT2 state. The polarization effects have been found to be minor for the LE state, when the charge density redistribution upon transition is not dramatic. The difference between BioEFP and NP-BioEFP site energies is $0.02 \mathrm{eV}$ and is independent of the ATP binding (Fig. 2).

\subsection{Electronic-Nuclear Interactions for Photoinduced Electron Transfer in Cryptochromes}

Electronic-nuclear interactions modulate the ultrafast PET processes, and need to be taken into account in dynamics simulations. Considering significant charge-density redistribution upon excitation from the ground to $\mathrm{CT} 1 / \mathrm{CT} 2$ states and the inhomogeneously charged protein environment, one can expect that both intra-fragment and intermolecular nuclear degrees of freedom would significantly alter the population dynamics. Below, we discuss the effects of the electronic system-bath couplings on the population dynamics upon PET in the cryptochrome protein.

The spectral densities for the intra-fragment bath, including the bath of nuclear modes of the quantum part, are shown in Figs. 3A-C.

Spectral densities for the CT states exhibit notable features in the low frequency range (Figs. 3B and 3C), mostly corresponding to the slower interchromophore motion (flavintryptophan, flavin-adenine). This suggests their important role in PET dynamics. The stronger coupling to the low-frequency normal modes for CT states is also apparent from the resulting reorganization energies (Eq. 4): the reorganization energies are 3-5 times larger for the CT states comparing to the LE state (Table 2). This difference in the values originates predominantly from contributions of the slow modes. It should be noted that the main features of intra-fragment spectral densities are not particularly sensitive to the presence of ATP. The position of peaks and their intensities are nearly identical. Indeed, the intrafragment reorganization energy does not change significantly due to binding of ATP for the 

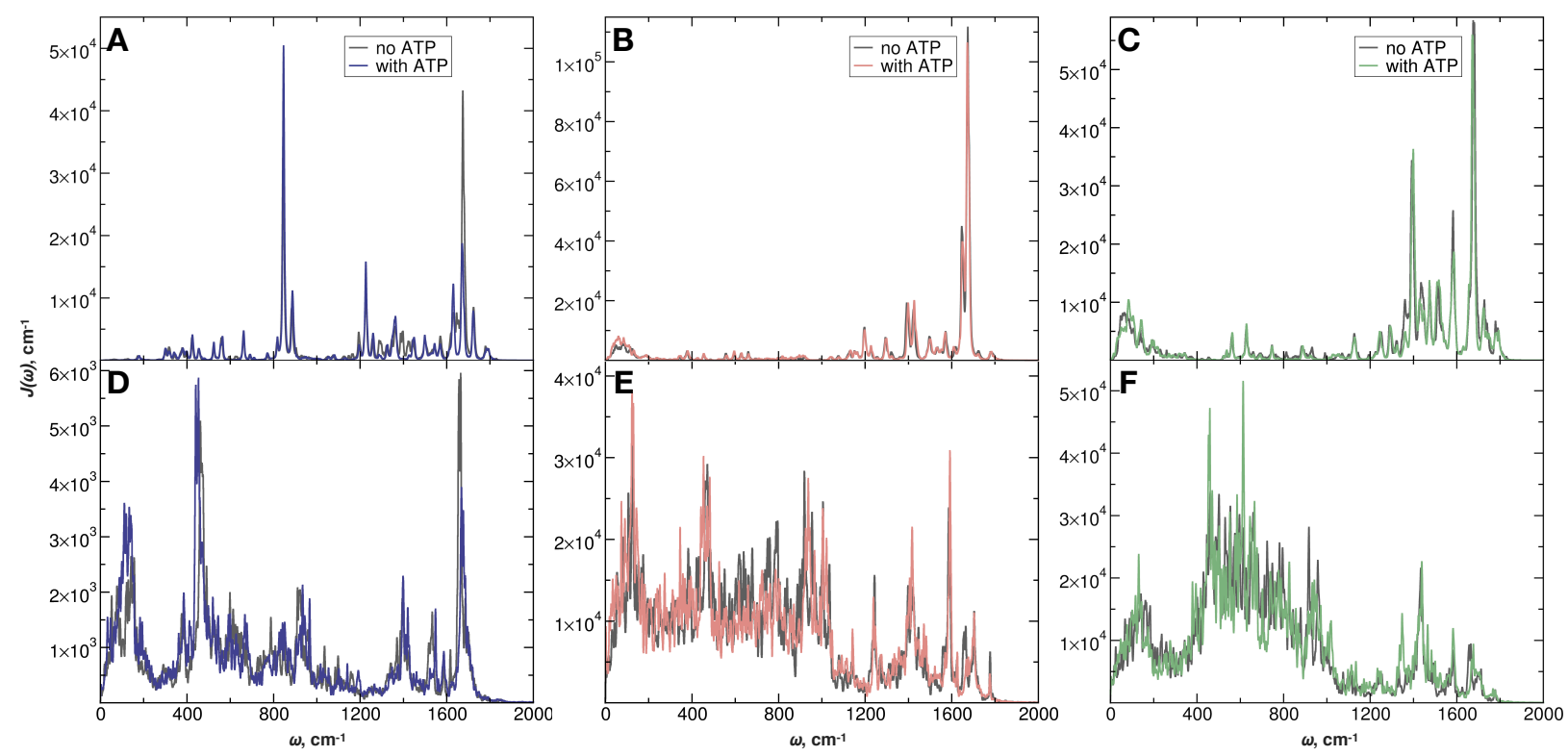

Figure 3: Spectral densities: intra-fragment component for (A) LE, (B) CT1, (C) CT2 states; intermolecular component for (D) LE, (E) CT1, (F) CT2 states.

LE and CT2 states: $0.13 \mathrm{eV}$ and $0.11 \mathrm{eV}$ for the LE state, $0.61 \mathrm{eV}$ and $0.64 \mathrm{eV}$ for the CT2 state in the absence and presence of ATP, respectively. The largest change has been observed for the CT1 state, the intra-fragment reorganization energy slightly increases in the presence of ATP: from $0.48 \mathrm{eV}$ to $0.62 \mathrm{eV}$.

The intermolecular nuclear bath is the bath of nuclear modes of the rest of the proteinsolvent environment, the remainder of the system that is not included in the QM part. Similar to their intra-fragment counterparts, intermolecular reorganization energies are found to be much larger for CT states than for the LE state (Table 2). This can be understood considering the different nature of the transitions. The charge density redistribution is located on one chromophore for LE state and is relatively minor. In contrast, excitation into CT states leads to dramatic charge redistribution causing a strong perturbation of the interactions of the CT state chromophores with their environment, and, therefore, results in larger reorganization energies. The intermolecular reorganization energies for the LE and CT2 states are not particularly sensitive to the presence of ATP: $0.20 \mathrm{eV}$ and $0.25 \mathrm{eV}$ for the LE state, $2.15 \mathrm{eV}$ and $2.24 \mathrm{eV}$ for the $\mathrm{CT} 2$ state in the absence and presence of ATP, respectively. However, the 
intermolecular reorganization energy for the CT1 state increases dramatically, when ATP is present: from $2.39 \mathrm{eV}$ to $3.07 \mathrm{eV}$.

The total reorganization energies (Table 2) for the CT states are larger by an order of magnitude comparing to the LE state. The reorganization energy for the LE state is not particularly sensitive to the presence of ATP $(+0.03 \mathrm{eV})$. The value for the CT2 state changes only by $0.12 \mathrm{eV}$. The reorganization energy for the CT1 state is strongly affected by the binding of ATP: the value in the presence of ATP is $0.82 \mathrm{eV}$ greater than in its absence.

It is important to note that the reorganization energies reported here correspond to a locally harmonic estimate of the energy drop from the FC point on a given excited state surface, to the equilibrium configuration in that particular excited state averaged over our ensemble. Thus, for example (see free energy estimates presented in Fig. S2), without the ATP we estimate that the LE state is $2.91 \mathrm{eV}$ above the FC point on the ground state and when the environment relaxes to the new equilibrium geometry in this LE state the energy, on average, drops slightly by $0.33 \mathrm{eV}$. The CT1 state under these conditions lies a little higher at the FC point with an excitation energy of $2.99 \mathrm{eV}$. However, the minimum on this CT1 state involves significant reorganization of the local environment so that the energy drops to the new CT1 state minimum energy geometry which is estimated to be $2.87 \mathrm{eV}$ suggesting that the equilibrium geometry in this $\mathrm{CT} 1$ state is on average only $0.12 \mathrm{eV}$ above the neutral ground state equilibrium geometry. In the absence of ATP the reorganization energy in the CT2 state $(2.76 \mathrm{eV})$ is comparable to that in $\mathrm{CT} 1$, so with the larger FC excitation energy to CT2 $(4.08 \mathrm{eV})$ this puts the energy of the CT2 state equilibrium configuration at $1.32 \mathrm{eV}$ above that of the ground state on average.

Interestingly, the very large reorganization energy we estimate in the CT1 state in the presence of ATP, some $3.69 \mathrm{eV}$, together with the CT1 FC excitation energy of $2.98 \mathrm{eV}$ under these conditions, suggests that the CT1 state average energy minimum in the presence of ATP actually lies at $-0.71 \mathrm{eV}$, i.e. below the average energy of the neutral equilibrium state.

There are large reorganization energy barriers between these minima on the different 
surfaces, and a more detailed understanding of the long time relaxation to equilibrium after photo-excitation must include a treatment of non-radiative relaxation and non-adiabatic coupling between these states. The early time PET processes studied in the next sections must thus involve exploration of metastable, non-equilibrium state dynamics that occurs well before equilibration after photoexcitation.

Table 2: Total reorganization energy $\left(\lambda_{\text {total }}\right.$, in $\left.\mathrm{eV}\right)$ and contributions computed for intrafragment $\left(\lambda_{\text {intra }}\right)$ and intermolecular $\left(\lambda_{\text {inter }}\right)$ baths for configurations without and with ATP

\begin{tabular}{cccc}
\hline \hline & $\lambda_{\text {intra }}$ & $\begin{array}{c}\lambda_{\text {inter }} \\
\text { LE }\end{array}$ & $\lambda_{\text {total }}$ \\
\hline without ATP & 0.13 & 0.20 & $\mathbf{0 . 3 3}$ \\
with ATP & 0.11 & 0.25 & $\mathbf{0 . 3 6}$ \\
\hline & & CT1 & \\
without ATP & 0.48 & 2.39 & $\mathbf{2 . 8 7}$ \\
with ATP & 0.62 & 3.07 & $\mathbf{3 . 6 9}$ \\
\hline & & CT2 & \\
without ATP & 0.61 & 2.15 & $\mathbf{2 . 7 6}$ \\
with ATP & 0.64 & 2.24 & $\mathbf{2 . 8 8}$ \\
\hline
\end{tabular}

\subsection{Effects of Polarization and Electronic-Nuclear Interactions on Population Dynamics}

Once the parameters for the generalized LE/CT state system-bath model Hamiltonian are obtained, one can proceed to simulating the dynamics of PET. Here, we only focus in detail on the case when ATP is present as the general trends in dynamics with and without ATP are very similar. The results of the dynamics simulations without ATP are provided in Supporting Information (Sec. S6).

We first consider the case of the isolated electronic system (system-bath interactions are turned off). As follows from Fig. 4A, there is no population transfer from the LE state to CT1 or CT2 in the case of purely electrostatic embedding (NP-BioEFP). This can be explained by the large energy gaps between the states: CT1 and CT2 are 1.21 and $1.06 \mathrm{eV}$ above the LE state, respectively (Fig. 2). Taking into account the environment polarization makes the CT1 
state almost degenerate with the LE state (0.08 eV gap) and enables some population transfer between the states (Fig. 2). However, the CT2 state is still not populated in the absence of the nuclear bath as the energy gap between the LE and CT2 states remains much larger (Fig. 2) comparing to the electronic couplings (Table 1). The small amplitude oscillations observed in these populations arise from persistent electronic coherent superposition states produced by the dynamics. These oscillations remain as they are not entirely washed out by the distribution of site disorder sampled in the ensemble.

We next analyze the effects of system-bath couplings on the CT dynamics (Fig. 4B, Fig. 4C) by considering either the intra-fragment or intermolecular bath coupled to the system. Interestingly, there are no oscillations anymore between the electronic states in population dynamics trajectories in both cases, and the dynamics becomes purely dissipative. The energy is being transferred between the electronic and nuclear subsystems, and the population of the LE state decays exponentially. Now the dynamical fluctuations incorporated through the different spectral densities and the explicit normal mode bath degrees of freedom cause rapid electronic decoherence so no oscillatory features survive ensemble averaging.

As follows from Fig. 4, turning on the system-bath interactions leads to the population transfer from the LE state to both CT1 and CT2 states. Interestingly, despite the large energy gap between the LE and CT2 states at the FC point, incorporating the bath degrees of freedom into the model and allowing relaxation in these coordinates enables significant population transfer to the CT2 state. Moreover, the population transfer to CT2 is more efficient than to CT1 in the presence of electronic-nuclear interactions. To summarize, the results point to the key role of electronic-nuclear interactions and bath relaxation dynamics in driving the PET in Cry1At-PHR.

While incorporating different parts of the bath (intra- and inter- Fig. 4B and C, respectively) does not change the picture qualitatively, the effects on the population transfer dynamics are pronounced (Fig. 4A). The detailed analysis of the time scales and relation of the computed quantities to the experimental data are discussed below. 

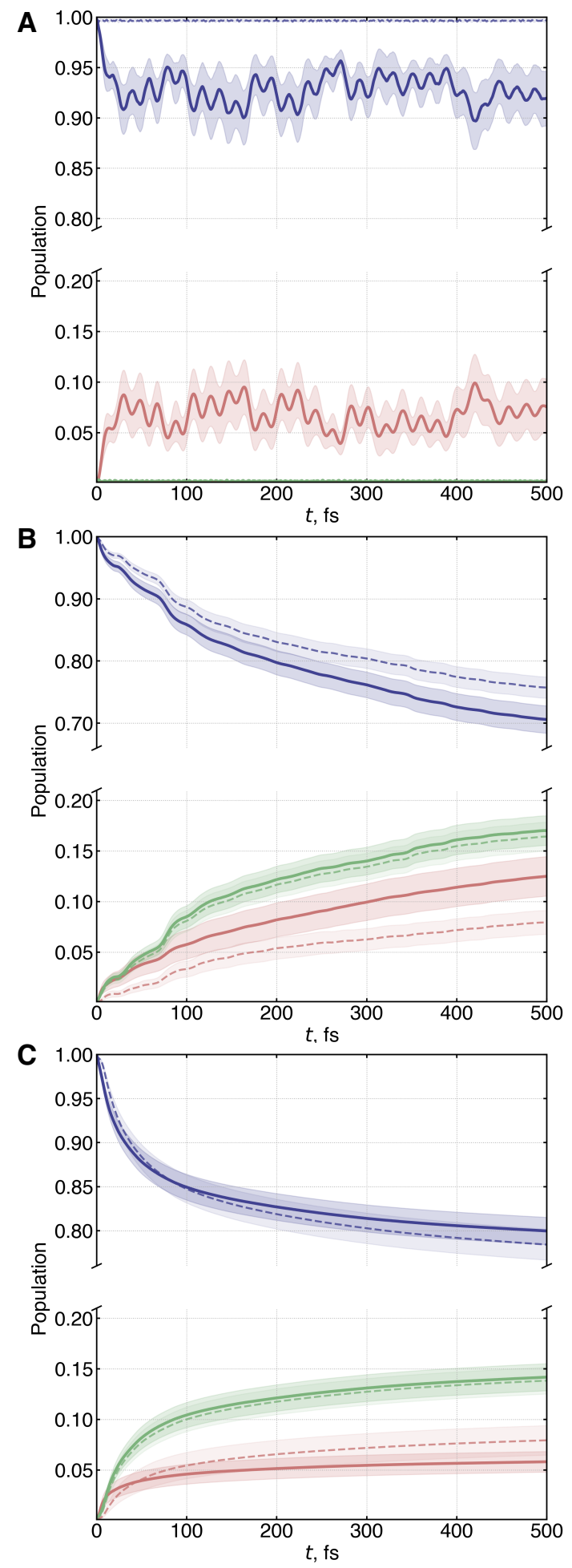

$\longrightarrow \mathrm{LE} \quad \mathrm{CT} 1-\mathrm{CT} 2$

Figure 4: Population dynamics for the first step of PET in Cry1At-PHR with (solid, BioEFP) and without (dash, NP-BioEFP) polarizable embedding obtained by averaging over 50 configurations with ATP being present: (A) no nuclear bath, (B) intra-fragment bath only, (C) intermolecular bath only. The statistical uncertainties are translucent. 


\subsection{Theoretical Rate Constants and Quantum Yields in Relation to Experiment}

The data obtained on state population evolution were fitted with the kinetic model shown in Fig. 5. Considering that the electronic couplings between CT1 and CT2 are much smaller than those for LE-CT1 and LE-CT2, direct transitions between CT1 and CT2 were neglected. The resulting rate constants are listed in Table 3. Note that the direct comparison of these calculated rate constants with the experimental estimates requires careful analysis. In particular, population of CT1 and CT2 states corresponds to formation of the tryptophan and adenine radical cations, respectively. Importantly, the absorption spectra of tryptophan and adenine radical cations overlap significantly in the UV/Vis region: both have the first peak at around $330 \mathrm{~nm}$ and a broad second peak with lower intensity in the range from 500 to $650 \mathrm{~nm}$. The absorption spectrum of the adenine radical cation has been recently reported by Markovitsi, Improta and coworkers; ${ }^{117}$ the spectrum of the tryptophan radical cation can be found in the work by Solar et al. ${ }^{118}$ As a result of the significant spectral overlap, it can be hard, to impossible, to unambiguously distinguish the individual electron transfer processes just based on the optical properties of the key species. One of the possible solutions to disentangle these two channels can be provided by time-resolved spectroscopy on mutants, in which the first tryptophan in the tryptophan triad, W400, is replaced by an ET inactive amino acid, e.g. phenylalanine or alanine. These studies would allow direct evaluation of the rate constant for the CT2 channel. However, such experimental data are not available for Cry1At-PHR. Importantly, despite the fact that ET from adenine to flavin of FAD has been most often disregarded in the analysis of the experimental results of Cry1At-PHR, this process is relatively fast in water ${ }^{48-51}(\approx 4 \mathrm{ps})$ and $\mathrm{D}_{2} \mathrm{O}^{52}(1.1 \mathrm{ps})$. Fast electron transfer between flavin and adenine of FAD has been mentioned earlier for plant Cryptochrome 3 from Arabidopsis thaliana. ${ }^{119}$ The characteristic lifetime of $1.4 \pm 0.4$ ps for this process was estimated for Drosophila melanogaster cryptochrome based on time-resolved fluorescent studies. ${ }^{120}$ Therefore, while there are no direct experimental data on the relevance 
of this process for Cry1At-PHR, indirect evidence available for other systems suggests that the PET from adenine to flavin can occur on the same timescale as the one from W400 to flavin, and therefore, this process must be taken into account for mechanistic description of photoactivation of cryptochromes.
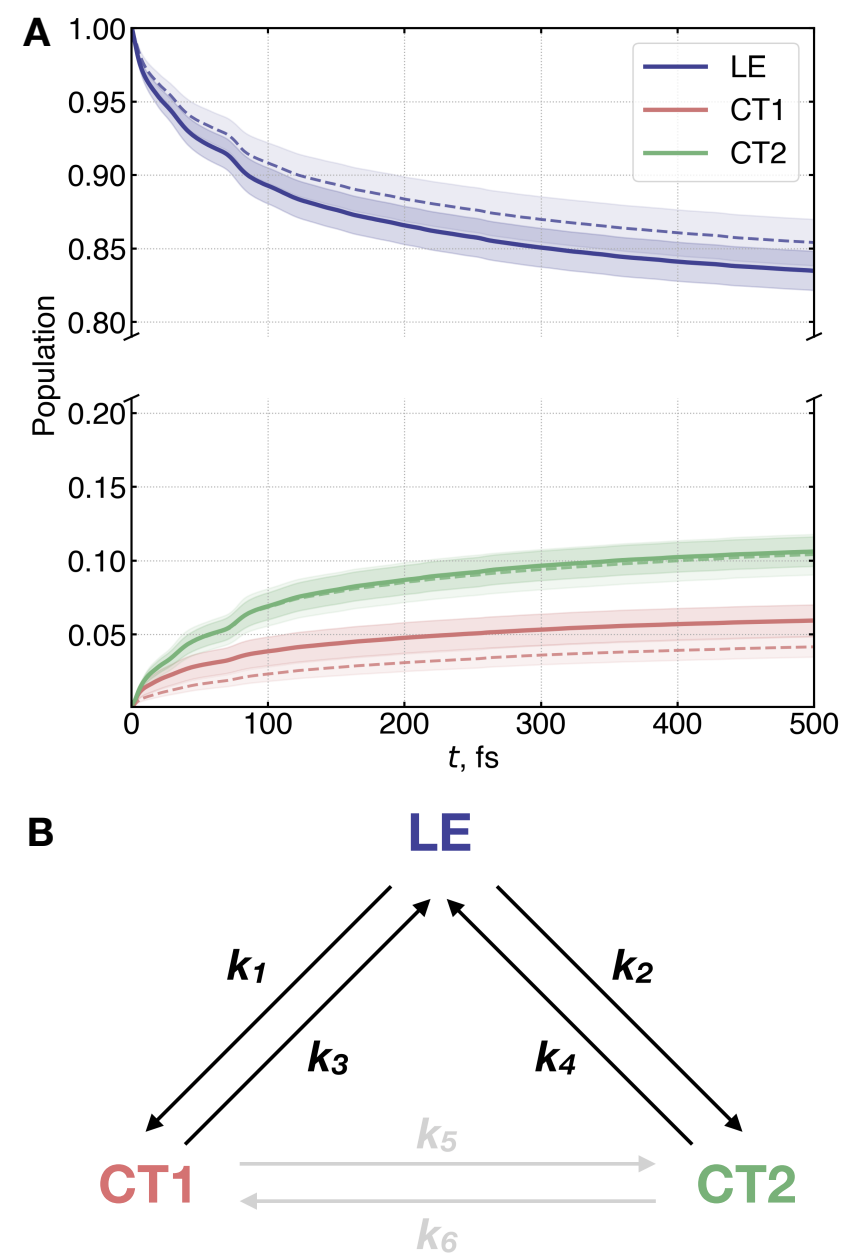

Figure 5: (A) Population dynamics for the first step of PET in Cry1At-PHR with (solid) and without ATP (dash) obtained by averaging over 50 configurations. The statistical errors are translucent. (B) PET kinetic model. Transitions between the CT1 and CT2 electronic states are omitted.

Overall, comparing to the available experimental time constants of the ultrafast PET and considering spectral indistinguishability of tryptophan and adenine radical cations, we sum up the two rate constants $k_{1}$ and $k_{2}$ (Fig. 5) to obtain the effective lifetime of the LE state: $\tau=\frac{1}{k_{1}+k_{2}}$. The rate constant estimates obtained in simulations without ATP are used 
for comparison with the experiment as the experiment has been performed in vitro in the absence of ATP.

The dynamics results with the intermolecular bath only (Fig. 4C) show that the CT2 state is being populated, yet the rate constants $k_{1}$ and $k_{2}$ for the CT1 and CT2 channels (Fig. 5) are nearly identical: $k_{1}^{\text {inter }}=2.2 \mathrm{ps}^{-1}$ vs. $k_{2}^{\text {inter }}=2.0 \mathrm{ps}^{-1}$. However, for the dynamics with the intra-fragment bath (Fig. 4B), the difference in the rate constants for LE $\rightarrow$ CT1 and LE $\rightarrow$ CT2 processes becomes significant $\left(k_{1}^{\text {intra }}=0.69 \mathrm{ps}^{-1}\right.$ vs. $\left.k_{2}^{\text {intra }}=1.1 \mathrm{ps}^{-1}\right)$. The greater rate constant for LE $\rightarrow \mathrm{CT} 2$ population transfer is also observed for dynamics with the full (intra-fragment + intermolecular) bath (Table 3). One can conclude that the system-bath interactions and a greater LE-CT2 electronic coupling value are the factors that are responsible for a more efficient population transfer to CT2 in comparison to CT1.

Bound ATP affects several parameters of the model Hamiltonian. It decreases the site energy of the CT2 state by $0.26 \mathrm{eV}$ as discussed above, which should favor population transfer to CT2. The site energy of the CT1 state does not change significantly. At the same time, bound ATP increases the electronic couplings between the LE and CT1 states, and leads to the greater value of the reorganization energy for the CT1 state. Yet, the LE-CT2 electronic couplings and CT2 reorganization energy do not change dramatically. Both larger reorganization energy and greater LE-CT1 coupling in the presence of ATP can promote population transfer to the CT1 state. Indeed, for the dynamics with the entire bath (Fig. 5), addition of ATP increases efficiency of the conventional ET channel: $k_{1}$ increases by a factor of two, from $0.31 \mathrm{ps}^{-1}$ to $0.66 \mathrm{ps}^{-1}$ (Table 3). The increase can be attributed to a greater electronic coupling, lower energy and larger reorganization energy in the presence of ATP (Tables 1 and 2). Adding ATP does not affect the LE-CT2 electronic couplings or reorganization energies, however it brings the CT2 energy significantly lower (Fig. 2A). Yet, the resulting $k_{2}$ rate constant is rather insensitive to the presence of ATP: $1.10 \mathrm{ps}^{-1}$ and $1.13 \mathrm{ps}^{-1}$ with and without ATP, respectively (Table 3). Importantly, the rate constants for LE $\rightarrow$ CT1 and $\mathrm{LE} \rightarrow \mathrm{CT} 2$ are of comparable magnitude. Thus, assuming further electron transfer from 
adenine of FAD to adenine of ATP, binding of ATP can enable an efficient PET channel that can explain the experimentally observed higher yield of the semireduced form of FAD in the presence of ATP. ${ }^{61}$ This effect has been previously explained by an increase in $\mathrm{p} K_{\mathrm{a}}$ of D396 aspartic acid in the presence of ATP (from 7.4 to > 9) ${ }^{61,62}$ However, it was not supported by the experimental studies on the cryptochrome from Chlamydomonas reinhardtii by Kottke and coworkers, ${ }^{60}$ where the authors showed that D396 was protonated in the dark irrespective of binding of ATP in the range of $\mathrm{pH}$ from 7.1 to 8.3. Moreover, we note that the $\mathrm{p} K_{\mathrm{a}}$ change hypothesis is unable to explain the recent UV/Vis and EPR experiments with the cryptochrome mutants, in which the main electron transfer pathway through the tryptophan triad is blocked. It was shown that semireduced FAD was formed in these mutants in vivo and in vitro in the presence of cellular metabolites. ${ }^{63,64}$

To the best of our knowledge, the only theoretical study exploring LE $\rightarrow$ CT2 electron transfer is that by de la Lande and coworkers. ${ }^{62}$ The authors used Marcus theory to quantify rate constants of $\mathrm{LE} \rightarrow \mathrm{CT} 1$ and $\mathrm{LE} \rightarrow \mathrm{CT} 2$ processes. The results of that work place $\mathrm{LE} \rightarrow \mathrm{CT} 1$ (CT2) electron transfer into the picosecond regime $(2-50 \mathrm{ps})$, with a $\mathrm{LE} \rightarrow$ CT2 electron transfer reported to be much slower. ${ }^{62}$ The discrepancy between the results by de la Lande and coworkers ${ }^{62}$ and those reported in the present work can be due to the following reasons. First, ET in cryptochromes is ultrafast and comparable to, or faster than the electroelastic fluctuations of the environment (protein-water) interface. ${ }^{76}$ Hence, the significant fraction of such fluctuations are essentially frozen out on the time scale of the ET, ${ }^{76}$ which was not taken into account in Ref. 62 . The effective reorganization energy for the ultrafast PET is expected to be significantly smaller than that estimated from the linear response approximation, ${ }^{76,121-123}$ and this would alter the rate constant estimate. Indeed, if the parameters obtained in this work are used to compute Marcus theory rate constant for $\mathrm{LE} \rightarrow \mathrm{CT} 2$ process, the lifetime becomes at least an order of magnitude greater compared to that extracted from the kinetic model. Second, de la Lande and coworkers ${ }^{62}$ exploited electronic embedding QM/MM schemes to evaluate the free energies of CT processes. Here, 
we have shown that the MM environment polarization has a profound effect on the energetics of charge separation processes. Therefore, using nonpolarizable QM/MM schemes can lead to significant errors. Third, LE-CT1 and LE-CT2 electronic couplings were approximated by those for the ground state instead of LE for flavin. ${ }^{62}$ The validity of this approximation is discussed in Sec. 2 and S3 of Supporting Information: the couplings can change by a factor of five.

Below, we compare the computed rate constants and quantum yields with available experimental data. The computed time constant (690 fs, without ATP, see Table 3) for Cry1AtPHR is in line with the experimental values reported independently for cryptochrome from Chlamydomonas reinhardtii (400-480 fs) ${ }^{47,81}$ and two flavoproteins of the cryptochrome photolyase family from the green algae Ostreococcus tauri (390-590 fs). ${ }^{124}$ Note that the absorption spectra of adenine and the adenine radical cation have not been included in the experimental analysis, and the fs-ps time-resolved spectroscopy experiments have been conducted on the different proteins, not on the one from Arabidopsis thaliana used in our simulations.

Table 3: Electron transfer rate (in $\mathrm{ps}^{-1}$ ) and time (in ps) constants computed from nonadiabatic dynamics simulations based on the populations of corresponding diabatic states

\begin{tabular}{cccc}
\hline \hline & with ATP & without ATP & experiment \\
\hline$k_{1}(\mathrm{LE} \rightarrow \mathrm{CT} 1)$ & 0.66 & 0.31 & - \\
$k_{2}(\mathrm{LE} \rightarrow \mathrm{CT} 2)$ & 1.10 & 1.13 & - \\
$k_{3}(\mathrm{CT} 1 \rightarrow \mathrm{LE})$ & 9.93 & 6.43 & - \\
$k_{4}(\mathrm{CT} 2 \rightarrow \mathrm{LE})$ & 9.08 & 9.84 & - \\
\hline$\tau(\mathrm{LE} \rightarrow(\mathrm{CT} 1+\mathrm{CT} 2))$ & 0.57 & 0.69 & $0.40-0.48^{a}, 0.39-0.59^{b}$ \\
\hline
\end{tabular}

${ }^{a}$ From Ref. 81, obtained for cryptochrome from Chlamydomonas reinhardtii

${ }^{b}$ From Ref. 124, obtained for flavoprotein of cryptochrome photolyase family from Ostreococcus tauri

The radical pair quantum yields have been computed by taking the magnitudes of populations of the corresponding CT states after 0.5 ps PLDM simulation. In the presence of ATP, the CT1 yield is increased by $1.7 \%$ (Table 4 ), while the CT2 yield does not change significantly. To compare the computed quantum yields to the experimental values, the fol- 
Table 4: Computed quantum yield (in \%) after 0.5 ps nonadiabtic dynamics simulations averaged over 50 configurations.

\begin{tabular}{ccc}
\hline \hline & without ATP & with ATP \\
\hline LE $\rightarrow$ CT1 & $4.2 \pm 0.7$ & $5.9 \pm 1.1$ \\
LE $\rightarrow$ CT2 & $10.4 \pm 1.4$ & $10.6 \pm 1.0$ \\
\hline $\mathrm{LE} \rightarrow($ CT1 + CT2 $)$ & $4.2 \pm 0.7(14.6 \pm 1.6)$ & $16.5 \pm 1.5$ \\
\hline
\end{tabular}

lowing considerations have been taken into account. First, both channels can contribute to the overall radical pair yield in the presence of ATP. Due to the substantial spectral overlap between tryptophan and adenine radical cations (for both protonated and unprotonated forms), CT1 and CT2 states become spectrally indistinguishable. Second, although the experimental quantum yield values were reported for the initial time, $t=0$ ns (see SI of Ref. 61), the time resolution was in sub-nanosecond - nanosecond range. This time is sufficient for the radical pair recombination processes to occur. Indeed, the reported backward ET time constant for structurally similar photolyases is about 100 ps. ${ }^{53-55}$ This can be particularly important for the CT2 radical pair (flavin-adenine) as the experiments were done on the protein without bound ATP, ${ }^{61}$ and the further steps of the proposed competing electron transfer channels were blocked. Therefore, the experimental total quantum yield likely does not contain a contribution from the CT2 in the absence of a possible hole acceptor, ATP. Third, given the time resolution of the experiment, the electron can hop further to other sites, not necessarily with close to $100 \%$ efficiency, and our simulations are only focused on the ultrafast PET, where the electron transfer and subsequent hole transfer are branching out. The total quantum yields taking into account the points above are reported in Table 4 . The value of $14.6 \pm 1.6 \%$ for $\mathrm{LE} \rightarrow(\mathrm{CT} 1+\mathrm{CT} 2)$ corresponds to the sum of quantum yields for CT1 and CT2, while $4.2 \pm 0.7 \%$ only accounts for the contribution from CT1. The discussion above suggests that the latter value should be treated as the best estimate based on the rate constants obtained in the current work. Therefore, the best estimates of the total quantum yields in the absence of ATP and presence of ATP are $4.2 \pm 0.7 \%$ and 16.5 $\pm 1.5 \%$, respectively. The computed total quantum yields are in excellent agreement with 
the experimental values of $6.9 \%$ and $19.9 \%$ by Müller et al. ${ }^{61}$

Finally, we point out that existence of the competing ET pathway brings a new perspective on the candidates for a signaling radical pair, in particular in the presence of cellular metabolites in vivo. The competing ET pathway discussed in this work, opens the possibility for the adenyl radical of ATP to be a part of the signaling radical pair or to be involved in its formation through a chain of subsequent ET processes. In addition, Y402 and Y399, $\pi$-stacked with the ATP adenine in Cry1At-PHR ${ }^{64}$ and Cry2At, ${ }^{63}$ respectively, can possibly serve as a terminal hole acceptor and be a part of the signaling radical pair alongside with the flavin moiety of FAD. Broader theoretical and experimental research on elucidating timescales of the branching PET pathways is needed to unravel the mechanistic details of photoactivation of cryptochrome proteins and answer the question whether cryptochrome proteins are responsible for sensing geomagnetic field by living organisms.

\section{Conclusions}

We present the results of the first-principles study of the molecular mechanism of photoactivation of cryptochromes, providing both accurate energetic and dynamical descriptions of the process. The theoretical rate constants as well as quantum yield have been obtained by using a multi-level approach, exploiting hybrid QM/MM methods with a nonempirical polarizable force field (BioEFP) and nonadiabatic semiclassical path integral dynamics (PLDM). The results show that photoexcitation can initiate two competing electron transfer channels and explain the enhanced yield of the semireduced form of FAD in the presence of ATP. The electron transfer rate constants and the estimates of the quantum yields are consistent with the available experimental data on cryptochromes in the presence and absence of ATP in vitro and in vivo. The results of this work contribute to the understanding of complex

photoinduced electron transfer processes accompanying formation of a photoactivated state of the cryptochrome proteins. 


\section{Acknowledgement}

This work was supported by Boston University, Department of Chemistry, and in part through computational resources provided by Boston University Shared Computing Cluster. R.N.T. and K.B.B. would like to thank Prof. Shirin Faraji (University of Groningen) for providing Q-Chem/GROMACS interface adapted for this work. R.N.T. and K.B.B. also thank Dr. MiKyung Lee for her help with initial setup of PLDM simulations. D.F.C. acknowledges support for this research from the National Science Foundation under Grant No. CHE-1665367. R.N.T. and J.P. acknowledge support from the Molecular Sciences Software Institute under Grant No. ACI-1547580.

\section{Supporting Information Available}

The Supporting Information is available free of charge on the ACS Publications website at DOI: .

Analysis of the crystal structure of Cry1At-PHR (PDB: $1 \mathrm{U}^{2} \mathrm{D}^{83}$ ) with eMap. ${ }^{125} \mathrm{GMH}$ electronic couplings computed using two- and three-state approximation employing various methods and schemes. Ensemble-averaged electronic Hamiltonian for configurations with and without ATP with the site energies computed using non-polarizable (NP-BioEFP) and polarizable (BioEFP) environment. Free energy estimates with respect to the ground state for the LE, CT1, and CT2 states. Population dynamics in the absence of ATP. Estimated electron transfer rate constants for configurations with and without ATP. Details on broadening used for constructing the spectral densities for the intra-fragment electronic-nuclear component. 


\section{References}

(1) Gust, D.; Moore, T. A.; Moore, A. L. Mimicking Photosynthetic Solar Energy Transduction. Acc. Chem. Res. 2001, 34, 40-48.

(2) Sinha, R. P.; Häder, D.-P. UV-induced DNA damage and repair: A review. Photochem. Photobiol. Sci. 2002, 1, 225-236.

(3) Foyer, C. H.; Noctor, G. Redox Regulation in Photosynthetic Organisms: Signaling, Acclimation, and Practical Implications. Antioxid. Redox Signal. 2009, 11, 861-905.

(4) Gust, D.; Moore, T.; Moore, A. Solar fuels via artificial photosynthesis. Acc. Chem. Res. 2009, 42, 1890-1898.

(5) Chaves, I.; Pokorny, R.; Byrdin, M.; Hoang, N.; Ritz, T.; Brettel, K.; Essen, L.O.; van der Horst, G. T. J.; Batschauer, A.; Ahmad, M. Cryptochromes: Blue light photoreceptors in plants and animals. Annu. Rev. Plant Biol. 2011, 62, 335-364.

(6) Tachibana, Y.; Vayssieres, L.; Durrant, J. R. Artificial photosynthesis for solar watersplitting. Nat. Photonics 2012, 6, 511-518.

(7) Bialas, C.; Jarocha, L. E.; Henbest, K. B.; Zollitsch, T. M.; Kodali, G.; Timmel, C. R.; Mackenzie, S. R.; Dutton, P. L.; Moser, C. C.; Hore, P. J. Engineering an Artificial Flavoprotein Magnetosensor. J. Am. Chem. Soc. 2016, 138, 16584-16587.

(8) Grayson, K. J.; Anderson, J. L. R. Designed for life: biocompatible de novo designed proteins and components. J. Royal Soc. Interface 2018, 15, 20180472.

(9) Mullineaux, P.; Karpinski, S. Signal transduction in response to excess light: getting out of the chloroplast. Curr. Opin. Plant Biol. 2002, 5, 43-48.

(10) Surpin, M.; Larkin, R. M.; Chory, J. Signal Transduction between the Chloroplast and the Nucleus. Plant Cell 2002, 14, S327-S338. 
(11) Apel, K.; Hirt, H. REACTIVE OXYGEN SPECIES: Metabolism, Oxidative Stress, and Signal Transduction. Annu. Rev. Plant Biol. 2004, 55, 373-399.

(12) Weber, S. Light-driven enzymatic catalysis of DNA repair: a review of recent biophysical studies on photolyase. Biochim. Biophys. Acta-Bioenergetics 2005, 1707, 1-23.

(13) Brettel, K.; Byrdin, M. Reaction mechanisms of DNA photolyase. Curr. Opin. Struct. Biol. 2010, 20, 693-701.

(14) Sherrard, R. M.; Morellini, N.; Jourdan, N.; El-Esawi, M.; Arthaut, L.-D.; Niessner, C.; Rouyer, F.; Klarsfeld, A.; Doulazmi, M.; Witczak, J.; d'Harlingue, A.; Mariani, J.; Mclure, I.; Martino, C. F.; Ahmad, M. Low-intensity electromagnetic fields induce human cryptochrome to modulate intracellular reactive oxygen species. PLOS Biol. 2018, 16, e2006229.

(15) Landler, L.; Keays, D. A. Cryptochrome: The magnetosensor with a sinister side? PLOS Biol. 2018, 16, e3000018.

(16) Qin, S.; Yin, H.; Yang, C.; Dou, Y.; Liu, Z.; Zhang, P.; Yu, H.; Huang, Y.; Feng, J.; Hao, J., et al, A magnetic protein biocompass. Nat. Mater. 2016, 15, 217-226.

(17) Schulten, K.; Swenberg, C.; Weller, A. A Biomagnetic Sensory Mechanism Based on Magnetic Field Modulated Coherent Electron Spin Motion. Zeitschrift für Physikalische Chemie 1978, 111, 1-5.

(18) Maeda, K.; Henbest, K.; Cintolesi, F.; Kuprov, I.; Rodgers, C.; Liddell, P.; Gust, D.; Timmel, C.; Hore, P. Chemical compass model of avian magnetoreception. Nature 2008, 453, 387-390.

(19) Efimova, O.; Hore, P. Role of exchange and dipolar interactions in the radical pair model of the avian magnetic compass. Biophys. J. 2008, 94, 1565-1574. 
(20) Biskup, T.; Schleicher, E.; Okafuji, A.; Link, G.; Hitomi, K.; Getzoff, E.; Weber, S. Direct observation of photoinduced radical pair in a cryptochrome blue-light photoreceptor. Angew. Chem. Int. Ed. 2009, 48, 404-407.

(21) Rodgers, C.; Hore, P. Chemical magnetoreception in birds: The radical pair mechanism. Proc. Nat. Acad. Sci. 2009, 106, 353-360.

(22) Hogben, H.; Efimova, O.; Wagner-Rundell, N.; Timmel, C.; Hore, P. Possible involvement of superoxide and dioxygen with cryptochrome in avian magnetoreception: Origin of Zeeman resonances observed by in vivo EPR spectroscopy. Chem. Phys. Lett. 2009, 480, 118-122.

(23) Solov'yov, I.; Schulten, K. Magnetoreception through cryptochrome may involve superoxide. Biophys. J. 2009, 96, 4804-4813.

(24) Ritz, T.; Wiltschko, R.; Hore, P.; Rodgers, C.; Stapput, K.; Thalau, P.; Timmel, C.; Wiltschko, W. Magnetic compass of birds is based on a molecule with optimal directional sensitivity. Biophys. J. 2009, 96, 3451-3457.

(25) Maeda, K.; Robinson, A. J.; Henbest, K. B.; Hogben, H. J.; Biskup, T.; Ahmad, M.; Schleicher, E.; Weber, S.; Timmel, C. R.; Hore, P. J. Magnetically sensitive lightinduced reactions in cryptochrome are consistent with its proposed role as a magnetoreceptor. Proc. Nat. Acad. Sci. 2012, 109, 4774-4779.

(26) Nießner, C.; Denzau, S.; Stapput, K.; Ahmad, M.; Peichl, L.; Wiltschko, W.; Wiltschko, R. Magnetoreception: activated cryptochrome 1a concurs with magnetic orientation in birds. J. Royal Soc. Interface 2013, 10, 20130638.

(27) Lee, A. A.; Lau, J. C. S.; Hogben, H. J.; Biskup, T.; Kattnig, D. R.; Hore, P. J. Alternative radical pairs for cryptochrome-based magnetoreception. J. Royal Soc. Interface 2014, 11, 20131063. 
(28) Hiscock, H. G.; Worster, S.; Kattnig, D. R.; Steers, C.; Jin, Y.; Manolopoulos, D. E.; Mouritsen, H.; Hore, P. J. The quantum needle of the avian magnetic compass. Proc. Nat. Acad. Sci. 2016, 113, 4634-4639.

(29) Kattnig, D. R.; Solov'yov, I. A.; Hore, P. J. Electron spin relaxation in cryptochromebased magnetoreception. Phys. Chem. Chem. Phys. 2016, 18, 12443-12456.

(30) Bouly, J.-P.; Schleicher, E.; Dionisio-Sese, M.; Vandenbussche, F.; Van Der Straeten, D.; Bakrim, N.; Meier, S.; Batschauer, A.; Galland, P.; Bittl, R.; Ahmad, M. Cryptochrome Blue Light Photoreceptors Are Activated through Interconversion of Flavin Redox States. J. Biol. Chem. 2007, 282, 9383-9391.

(31) Berndt, A.; Kottke, T.; Breitkreuz, H.; Dvorsky, R.; Hennig, S.; Alexander, M.; Wolf, E. A Novel Photoreaction Mechanism for the Circadian Blue Light Photoreceptor Drosophila Cryptochrome. J. Biol. Chem. 2007, 282, 13011-13021.

(32) Banerjee, R.; Schleicher, E.; Meier, S.; Viana, R. M.; Pokorny, R.; Ahmad, M.; Bittl, R.; Batschauer, A. The Signaling State of Arabidopsis Cryptochrome 2 Contains Flavin Semiquinone. J. Biol. Chem. 2007, 282, 14916-14922.

(33) Hoang, N.; Schleicher, E.; Kacprzak, S.; Bouly, J.-P.; Picot, M.; Wu, W.; Berndt, A.; Wolf, E.; Bittl, R.; Ahmad, M. Human and Drosophila Cryptochromes Are Light Activated by Flavin Photoreduction in Living Cells. PLOS Biol. 2008, 6, e160.

(34) Aubert, C.; Vos, M. H.; Mathis, P.; Eker, A. P.; Brettel, K. Intraprotein radical transfer during photoactivation of DNA photolyase. Nature 2000, 405, 586-590.

(35) Froy, O.; Chang, D. C.; Reppert, S. M. Redox Potential: Differential Roles in dCRY and mCRY1 Functions. Curr. Biol. 2002, 12, 147-152.

(36) Zeugner, A.; Byrdin, M.; Bouly, J.-P.; Bakrim, N.; Giovani, B.; Brettel, K.; Ah- 
mad, M. Light-induced Electron Transfer in Arabidopsis Cryptochrome-1 Correlates with in Vivo Function. J. Biol. Chem. 2005, 280, 19437-19440.

(37) Essen, L.-O. Photolyases and cryptochromes: Common mechanisms of DNA repair and light-driven signaling? Curr. Opin. Struct. Biol. 2006, 16, 51-59.

(38) Lüdemann, G.; Solov'yov, I. A.; Kubař, T.; Elstner, M. Solvent Driving Force Ensures Fast Formation of a Persistent and Well-Separated Radical Pair in Plant Cryptochrome. J. Am. Chem. Soc. 2015, 137, 1147-1156.

(39) Muller, P.; Yamamoto, J.; Martin, R.; Iwai, S.; Brettel, K. Discovery and functional analysis of a 4th electron-transferring tryptophan conserved exclusively in animal cryptochromes and (6-4) photolyases. Chem. Comm. 2015, 51, 15502-15505.

(40) Nohr, D.; Franz, S.; Rodriguez, R.; Paulus, B.; Essen, L.-O.; Weber, S.; Schleicher, E. Extended Electron-Transfer in Animal Cryptochromes Mediated by a Tetrad of Aromatic Amino Acids. Biophys. J. 2016, 111, 301-311.

(41) Giovani, B.; Byrdin, M.; Ahmad, M.; Brettel, K. Light-induced electron transfer in a cryptochrome blue-light photoreceptor. Nat. Struct. Mol. Biol. 2003, 10, 489-490.

(42) Liedvogel, M.; Maeda, K.; Henbest, K.; Schleicher, E.; Simon, T.; Timmel, C. R.; Hore, P. J.; Mouritsen, H. Chemical Magnetoreception: Bird Cryptochrome 1a Is Excited by Blue Light and Forms Long-Lived Radical-Pairs. PLOS ONE 2007, 2, e1106.

(43) Karogodina, T. Y.; Dranov, I. G.; Sergeeva, S. V.; Stass, D. V.; Steiner, U. E. Kinetic Magnetic-Field Effect Involving the Small Biologically Relevant Inorganic Radicals $\mathrm{NO}$ and $\mathrm{O}_{2}{ }^{--}$. ChemPhysChem 2011, 12, 1714-1728.

(44) Bednarek, J.; Plonka, A.; Hallbrucker, A.; Mayer, E.; Symons, M. C. R. Hydroperoxyl 
Radical Generation by $\gamma$-Irradiation of Glassy Water at $77 \mathrm{~K}$. J. Am. Chem. Soc. 1996, 118, 9387-9390.

(45) Mondal, P.; Huix-Rotllant, M. Theoretical insights into the formation and stability of radical oxygen species in cryptochromes. Phys. Chem. Chem. Phys. 2019, 21, 88748882.

(46) Tomkiewicz, M.; McAlpine, R. D.; Cocivera, M. Photooxidation and Decarboxylation of Tyrosine Studied by E.P.R. and C.I.D.N.P. Techniques. Can. J. Chem. 1972, 50, $3849-3856$.

(47) Lacombat, F.; Espagne, A.; Dozova, N.; Plaza, P.; Müller, P.; Brettel, K.; FranzBadur, S.; Essen, L.-O. Ultrafast Oxidation of a Tyrosine by Proton-Coupled Electron Transfer Promotes Light Activation of an Animal-like Cryptochrome. J. Am. Chem. Soc. 2019, 141, 13394-13409.

(48) Enescu, M.; Lindqvist, L.; Soep, B. Excited-State Dynamics of Fully Reduced Flavins and Flavoenzymes Studied at Subpicosecond Time Resolution. Photochem. Photobiol. 1998, 68, 150-156.

(49) Stanley, R. J.; MacFarlane, Ultrafast Excited State Dynamics of Oxidized Flavins: Direct Observations of Quenching by Purines. J. Phys. Chem. A 2000, 104, 68996906.

(50) Chosrowjan, H.; Taniguchi, S.; Mataga, N.; Tanaka, F.; Visser, A. J. The stacked flavin adenine dinucleotide conformation in water is fluorescent on picosecond timescale. Chem. Phys. Lett. 2003, 378, 354-358.

(51) Nakabayashi, T.; Islam, M. S.; Ohta, N. Fluorescence Decay Dynamics of Flavin Adenine Dinucleotide in a Mixture of Alcohol and Water in the Femtosecond and Nanosecond Time Range. J. Phys. Chem. B 2010, 114, 15254-15260. 
(52) Li, G.; Glusac, K. D. The Role of Adenine in Fast Excited-State Deactivation of FAD: a Femtosecond Mid-IR Transient Absorption Study. J. Phys. Chem. B 2009, 113, 9059-9061.

(53) Liu, Z.; Tan, C.; Guo, X.; Li, J.; Wang, L.; Sancar, A.; Zhong, D. Determining complete electron flow in the cofactor photoreduction of oxidized photolyase. Proc. Nat. Acad. Sci. 2013, 110, 12966-12971.

(54) Liu, Z.; Zhang, M.; Guo, X.; Tan, C.; Li, J.; Wang, L.; Sancar, A.; Zhong, D. Dynamic determination of the functional state in photolyase and the implication for cryptochrome. Proc. Nat. Acad. Sci. 2013, 110, 12972-12977.

(55) Liu, Z.; Tan, C.; Guo, X.; Li, J.; Wang, L.; Zhong, D. Dynamic Determination of Active-Site Reactivity in Semiquinone Photolyase by the Cofactor Photoreduction. J. Phys. Chem. Lett. 2014, 5, 820-825.

(56) Zhong, D.; Zewail, A. H. Femtosecond dynamics of flavoproteins: Charge separation and recombination in riboflavine (vitamin $\mathrm{B}_{2}$ )-binding protein and in glucose oxidase enzyme. Proc. Nat. Acad. Sci. 2001, 98, 11867-11872.

(57) Gauden, M.; van Stokkum, I. H. M.; Key, J. M.; Lührs, D. C.; van Grondelle, R.; Hegemann, P.; Kennis, J. T. M. Hydrogen-bond switching through a radical pair mechanism in a flavin-binding photoreceptor. Proc. Nat. Acad. Sci. 2006, 103, 1089510900.

(58) Bouly, J.-P.; Giovani, B.; Djamei, A.; Mueller, M.; Zeugner, A.; Dudkin, E. A.; Batschauer, A.; Ahmad, M. Novel ATP-binding and autophosphorylation activity associated with Arabidopsis and human cryptochrome-1. Eur. J. Biochem. 2003, 270, $2921-2928$.

(59) Burney, S.; Hoang, N.; Caruso, M.; Dudkin, E. A.; Ahmad, M.; Bouly, J.-P. Confor- 
mational change induced by ATP binding correlates with enhanced biological function of Arabidopsis cryptochrome. FEBS Lett. 2009, 583, 1427-1433.

(60) Schroeder, L.; Oldemeyer, S.; Kottke, T. Time-Resolved Infrared Spectroscopy on Plant Cryptochrome-Relevance of Proton Transfer and ATP Binding for Signaling. J. Phys. Chem. A 2018, 122, 140-147.

(61) Müller, P.; Bouly, J. P.; Hitomi, K.; Balland, V.; Getzoff, E. D.; Ritz, T.; Brettel, K. ATP Binding Turns Plant Cryptochrome Into an Efficient Natural Photoswitch. Sci. Rep. 2014, 4, 5175 .

(62) Cailliez, F.; Müller, P.; Gallois, M.; de la Lande, A. ATP Binding and Aspartate Protonation Enhance Photoinduced Electron Transfer in Plant Cryptochrome. J. Am. Chem. Soc. 2014, 136, 12974-12986.

(63) Engelhard, C.; Wang, X.; Robles, D.; Moldt, J.; Essen, L.-O.; Batschauer, A.; Bittl, R.; Ahmad, M. Cellular Metabolites Enhance the Light Sensitivity of Arabidopsis Cryptochrome through Alternate Electron Transfer Pathways. The Plant Cell 2014, 26, 4519-4531.

(64) El-Esawi, M.; Glascoe, A.; Engle, D.; Ritz, T.; Link, J.; Ahmad, M. Cellular metabolites modulate in vivo signaling of Arabidopsis cryptochrome-1. Plant Signal. Behav. 2015, 10,9 .

(65) Doust, A. B.; van Stokkum, I. H. M.; Larsen, D. S.; Wilk, K. E.; Curmi, P. M. G.; van Grondelle, R.; Scholes, G. D. Mediation of Ultrafast Light-Harvesting by a Central Dimer in Phycoerythrin 545 Studied by Transient Absorption and Global Analysis. J. Phys. Chem. B 2005, 109, 14219-14226.

(66) Novoderezhkin, V. I.; Doust, A. B.; Curutchet, C.; Scholes, G. D.; van Grondelle, R. Excitation Dynamics in Phycoerythrin 545: Modeling of Steady-State Spectra and Transient Absorption with Modified Redfield Theory. Biophys. J. 2010, 99, 344-352. 
(67) Hayes, D.; Engel, G. Extracting the Excitonic Hamiltonian of the Fenna-MatthewsOlson Complex Using Three-Dimensional Third-Order Electronic Spectroscopy. Biophys. J. 2011, 100, 2043-2052.

(68) Wlodarczyk, L.; Snellenburg, J.; Ihalainen, J.; van Grondelle, R.; van Stokkum, I.; Dekker, J. Functional Rearrangement of the Light-Harvesting Antenna upon State Transitions in a Green Alga. Biophys. J. 2015, 108, 261-271.

(69) Lee, M. K.; Huo, P.; Coker, D. F. Semiclassical Path Integral Dynamics: Photosynthetic Energy Transfer with Realistic Environment Interactions. Annu. Rev. Phys. Chem. 2016, 67, 639-668.

(70) Lee, M. K.; Coker, D. F. Modeling Electronic-Nuclear Interactions for Excitation Energy Transfer Processes in Light-Harvesting Complexes. J. Phys. Chem. Lett. 2016, 7, 3171-3178.

(71) Lee, M. K.; Bravaya, K. B.; Coker, D. F. First-Principles Models for Biological LightHarvesting: Phycobiliprotein Complexes from Cryptophyte Algae. J. Am. Chem. Soc. 2017, 139, 7803-7814.

(72) Li, X.; Parrish, R. M.; Liu, F.; Kokkila Schumacher, S. I. L.; Martínez, T. J. An Ab Initio Exciton Model Including Charge-Transfer Excited States. J. Chem. Theory Comput. 2017, 13, 3493-3504.

(73) Ianeselli, A.; Orioli, S.; Spagnolli, G.; Faccioli, P.; Cupellini, L.; Jurinovich, S.; Mennucci, B. Atomic Detail of Protein Folding Revealed by an Ab Initio Reappraisal of Circular Dichroism. J. Am. Chem. Soc. 2018, 140, 3674-3682.

(74) Li, X.; Tully, J. C.; Schlegel, H. B.; Frisch, M. J. Ab initio Ehrenfest dynamics. J. Chem. Phys. 2005, 123, 084106. 
(75) Tully, J. Molecular Dynamics with Electronic Transitions. J. Chem. Phys. 1990, 93, 1061-1071.

(76) Blumberger, J. Recent Advances in the Theory and Molecular Simulation of Biological Electron Transfer Reactions. Chem. Rev. 2015, 115, 11191-11238.

(77) Huo, P.; Coker, D. F. Communication: Partial linearized density matrix dynamics for dissipative, non-adiabatic quantum evolution. J. Chem. Phys. 2011, 135, 201101.

(78) Huo, P.; Coker, D. F. Consistent schemes for non-adiabatic dynamics derived from partial linearized density matrix propagation. J. Chem. Phys. 2012, 137, 22A535.

(79) Huo, P.; Coker, D. F. Semi-classical path integral non-adiabatic dynamics: a partial linearized classical mapping Hamiltonian approach. Mol. Phys. 2012, 110, 1035-1052.

(80) Tazhigulov, R. N.; Gurunathan, P. K.; Kim, Y.; Slipchenko, L.; Bravaya, K. B. Polarizable Embedding for Simulating Redox Potentials of Biomolecules. Phys. Chem. Chem. Phys. 2019, 21, 11642-11650.

(81) Immeln, D.; Weigel, A.; Kottke, T.; Lustres, J. L. P. Primary Events in the Blue Light Sensor Plant Cryptochrome: Intraprotein Electron and Proton Transfer Revealed by Femtosecond Spectroscopy. J. Am. Chem. Soc. 2012, 134, 12536-12546.

(82) Abraham, M. J.; Murtola, T.; Schulz, R.; Páll, S.; Smith, J. C.; Hess, B.; Lindahl, E. GROMACS: High performance molecular simulations through multi-level parallelism from laptops to supercomputers. SoftwareX 2015, 1-2, 19-25.

(83) Brautigam, C. A.; Smith, B. S.; Ma, Z.; Palnitkar, M.; Tomchick, D. R.; Machius, M.; Deisenhofer, J. Structure of the photolyase-like domain of cryptochrome 1 from Arabidopsis thaliana. Proc. Nat. Acad. Sci. 2004, 101, 12142-12147. 
(84) Caprasecca, S.; Jurinovich, S.; Viani, L.; Curutchet, C.; Mennucci, B. Geometry Optimization in Polarizable QM/MM Models: The Induced Dipole Formulation. J. Chem. Theory Comput. 2014, 10, 1588-1598.

(85) Rhee, Y.; Head-Gordon, M. Scaled second order perturbation corrections to configuration interaction singles: Efficient and reliable excitation energy methods. J. Phys. Chem. A 2007, 111, 5314-5326.

(86) Dunning, T. Gaussian basis sets for use in correlated molecular calculations. I. The atoms boron through neon and hydrogen. J. Chem. Phys. 1989, 90, 1007-1023.

(87) Chibani, S.; Laurent, A. D.; Le Guennic, B.; Jacquemin, D. Improving the Accuracy of Excited-State Simulations of BODIPY and Aza-BODIPY Dyes with a Joint SOSCIS(D) and TD-DFT Approach. J. Chem. Theory Comput. 2014, 10, 4574-4582.

(88) Kendall, R.; T.H. Dunning, J.; Harrison, R. Electron affinities of the first-row atoms revisited. Systematic basis sets and wavefunctions. J. Chem. Phys. 1992, 96, 67966806.

(89) Wu, Q.; Cheng, C.-L.; Van Voorhis, T. Configuration interaction based on constrained density functional theory: A multireference method. J. Chem. Phys. 2007, 127, 164119.

(90) Van Voorhis, T.; Kowalczyk, T.; Kaduk, B.; Wang, L.-P.; Cheng, C.-L.; Wu, Q. The Diabatic Picture of Electron Transfer, Reaction Barriers, and Molecular Dynamics. Annu. Rev. Phys. Chem. 2010, 61, 149-170.

(91) Chai, J.; Head-Gordon, M. Systematic optimization of long-range corrected hybrid density functionals. J. Chem. Phys. 2008, 128, 084106.

(92) Chai, J.; Head-Gordon, M. Long-range corrected hybrid density functionals with 
damped atom-atom dispersion interactions. Phys. Chem. Chem. Phys. 2008, 10, 66156620 .

(93) Hehre, W.; Ditchfield, R.; Pople, J. Self-consistent molecular orbital methods. XII. Further extensions of Gaussian-type basis sets for use in molecular orbital studies of organic molecules. J. Chem. Phys. 1972, 56, 2257-2261.

(94) Hariharan, P.; Pople, J. The influence of polarization functions on molecular orbital hydrogenation energies. Theor. Chim. Acta 1973, 28, 213.

(95) Clark, T.; Chandrasekhar, J.; Spitznagel, G. W.; Schleyer, P. Efficient diffuse functionaugmented basis sets for anion calculations. III. The $3-21+\mathrm{G}$ basis set for first-row elements, Li-F. J. Comput. Chem. 1983, 4, 294-301.

(96) Frisch, M.; Pople, J.; Binkley, J. Self-consistent molecular orbital methods 25. Supplementary functions for Gaussian basis sets. J. Chem. Phys. 1984, 80, 3265-3269.

(97) Shao, Y.; Gan, Z.; Epifanovsky, E.; Gilbert, A.T.B.; Wormit, M.; Kussmann, J.; Lange, A.W.; Behn, A.; Deng, J.; Feng, X., et al, Advances in molecular quantum chemistry contained in the Q-Chem 4 program package. Mol. Phys. 2015, 113, 184215.

(98) Day, P.; Jensen, J.; Gordon, M.; Webb, S.; Stevens, W.; Krauss, M.; Garmer, D.; Basch, H.; Cohen, D. An effective fragment method for modeling solvent effects in quantum mechanical calculations. J. Chem. Phys. 1996, 105, 1968-1986.

(99) Gordon, M.; Freitag, M.; Bandyopadhyay, P.; Jensen, J.; Kairys, V.; Stevens, W. The effective fragment potential method: A QM-based MM approach to modeling environmental effects in chemistry. J. Phys. Chem. A 2001, 105, 293-307.

(100) Ghosh, D.; Kosenkov, D.; Vanovschi, V.; Williams, C.; Herbert, J.; Gordon, M.; Schmidt, M.; Slipchenko, L.; Krylov, A. Non-covalent interactions in extended sys- 
tems described by the Effective Fragment Potential method: Theory and application to nucleobase oligomers. J. Phys. Chem. A 2010, 114, 12739-12745.

(101) Gordon, M. S.; Smith, Q. A.; Xu, P.; Slipchenko, L. V. Accurate First Principles Model Potentials for Intermolecular Interactions. Annu. Rev. Phys. Chem. 2013, 64, $553-578$.

(102) Gordon, M.; Fedorov, D.; Pruitt, S.; Slipchenko, L. Fragmentation Methods: A Route to Accurate Calculations on Large Systems. Chem. Rev. 2012, 112, 632-672.

(103) Gurunathan, P.; Acharya, A.; Ghosh, D.; Kosenkov, D.; Kaliman, I.; Shao, Y.; Krylov, A.; Slipchenko, L. Extension of the Effective Fragment Potential Method to Macromolecules. J. Phys. Chem. B 2016, 120, 6562-6574.

(104) Slipchenko, L. Solvation of the Excited States of Chromophores in Polarizable Environment: Orbital Relaxation versus Polarization. J. Phys. Chem. A 2010, 114, $8824-8830$.

(105) Kosenkov, D.; Slipchenko, L. Solvent Effects on the Electronic Transitions of pNitroaniline: A QM/EFP Study. J. Phys. Chem. A 2011, 115, 392-401.

(106) DeFusco, A.; Minezawa, N.; Slipchenko, L.; Zahariev, F.; Gordon, M. Modeling Solvent Effects on Electronic Excited States. J. Phys. Chem. Lett. 2011, 2, 2184-2192.

(107) Ghosh, D.; Isayev, O.; Slipchenko, L.; Krylov, A. The effect of solvation on vertical ionization energy of thymine: From microhydration to bulk. J. Phys. Chem. A 2011, 115, 6028-6038.

(108) Tentscher, P. R.; Seidel, R.; Winter, B.; Guerard, J. J.; Arey, J. S. Exploring the aqueous vertical ionization of organic molecules by molecular simulation and liquid microjet photoelectron spectroscopy. J. Phys. Chem. B 2015, 119, 238-256. 
(109) Guerard, J. J.; Tentscher, P. R.; Seijo, M.; Arey, J. S. Explicit solvent simulations of the aqueous oxidation potential and reorganization energy for neutral molecules: gas phase, linear solvent response, and non-linear response contributions. Phys. Chem. Chem. Phys. 2015, 17, 14811-14826.

(110) Tazhigulov, R. N.; Bravaya, K. B. Free Energies of Redox Half-Reactions from FirstPrinciples Calculations. J. Phys. Chem. Lett. 2016, 7, 2490-2495.

(111) Cave, R.; Newton, M. Calculation of electronic coupling matrix elements for ground and excited state electron transfer reactions: Comparison of the generalized MullikenHush and block diagonalization method. J. Chem. Phys. 1997, 106, 9213-9226.

(112) Foresman, J.; Head-Gordon, M.; Pople, J.; Frisch, M. Toward a systematic molecular orbital theory for excited states. J. Phys. Chem. 1992, 96, 135-149.

(113) Rust, M.; Lappe, J.; Cave, R. J. Multistate Effects in Calculations of the Electronic Coupling Element for Electron Transfer Using the Generalized Mulliken-Hush Method. J. Phys. Chem. A 2002, 106, 3930-3940.

(114) Bogdanov, A. M.; Acharya, A.; Titelmayer, A. V.; Mamontova, A. V.; Bravaya, K. B.; Kolomeisky, A. B.; Lukyanov, K. A.; Krylov, A. I. Turning On and Off Photoinduced Electron Transfer in Fluorescent Proteins by $\pi$-Stacking, Halide Binding, and Tyr145 Mutations. Journal of the American Chemical Society 2016, 138, 4807-4817.

(115) Bader, J. S.; Berne, B. J. Quantum and classical relaxation rates from classical simulations. J. Chem. Phys. 1994, 100, 8359-8366.

(116) Rivera, E.; Montemayor, D.; Masia, M.; Coker, D. F. Influence of Site-Dependent Pigment-Protein Interactions on Excitation Energy Transfer in Photosynthetic Light Harvesting. J. Phys. Chem. B 2013, 117, 5510-5521. 
(117) Banyasz, A.; Ketola, T.-M.; Muñoz-Losa, A.; Rishi, S.; Adhikary, A.; Sevilla, M. D.; Martinez-Fernandez, L.; Improta, R.; Markovitsi, D. UV-Induced Adenine Radicals Induced in DNA A-Tracts: Spectral and Dynamical Characterization. J. Phys. Chem. Lett. 2016, 7, 3949-3953.

(118) Solar, S.; Getoff, N.; Surdhar, P. S.; Armstrong, D. A.; Singh, A. Oxidation of tryptophan and $N$-methylindole by $\mathrm{N}_{3}, \mathrm{Br}_{2}{ }^{-}$, and $(\mathrm{SCN})_{2}{ }^{--}$radicals in light- and heavywater solutions: A pulse radiolysis study. J. Phys. Chem. 1991, 95, 3639-3643.

(119) Song, S.-H.; Dick, B.; Penzkofer, A.; Pokorny, R.; Batschauer, A.; Essen, L.-O. Absorption and fluorescence spectroscopic characterization of cryptochrome 3 from Arabidopsis thaliana. J. Photochem. Photobiol. B 2006, 85, 1-16.

(120) Shirdel, J.; Zirak, P.; Penzkofer, A.; Breitkreuz, H.; Wolf, E. Absorption and fluorescence spectroscopic characterisation of the circadian blue-light photoreceptor cryptochrome from Drosophila melanogaster (dCry). Chem. Phys. 2008, 352, 35-47.

(121) Matyushov, D. V. Protein electron transfer: Dynamics and statistics. J. Chem. Phys. 2013, 139, 025102 .

(122) Matyushov, D. V. Protein electron transfer: is biology (thermo)dynamic? J. Phys. Condens. Matter 2015, 27, 473001.

(123) LeBard, D. N.; Kapko, V.; Matyushov, D. V. Energetics and Kinetics of Primary Charge Separation in Bacterial Photosynthesis. J. Phys. Chem. B 2008, 112, 1032210342.

(124) Brazard, J.; Usman, A.; Lacombat, F.; Ley, C.; Martin, M. M.; Plaza, P.; Mony, L.; Heijde, M.; Zabulon, G.; Bowler, C. Spectro-Temporal Characterization of the Photoactivation Mechanism of Two New Oxidized Cryptochrome/Photolyase Photoreceptors. J. Am. Chem. Soc. 2010, 132, 4935-4945. 
(125) Tazhigulov, R. N.; Gayvert, J. R.; Wei, M.; Bravaya, K. B. eMap: A Web Application for Identifying and Visualizing Electron or Hole Hopping Pathways in Proteins. J. Phys. Chem. B 2019, 123, 6946-6951. 


\section{Graphical TOC Entry}

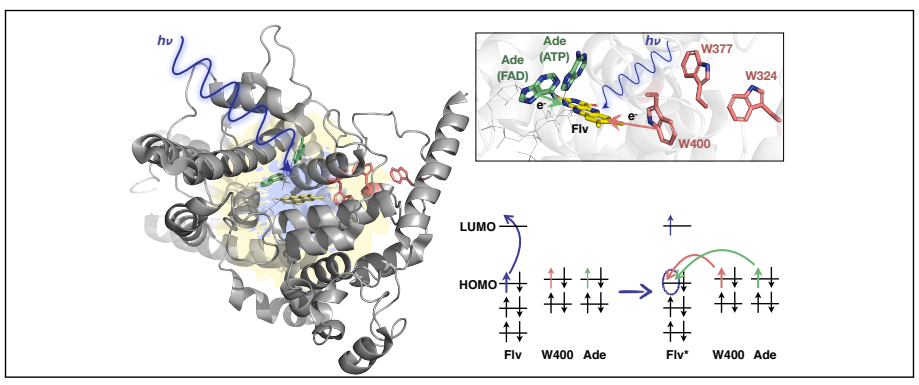

